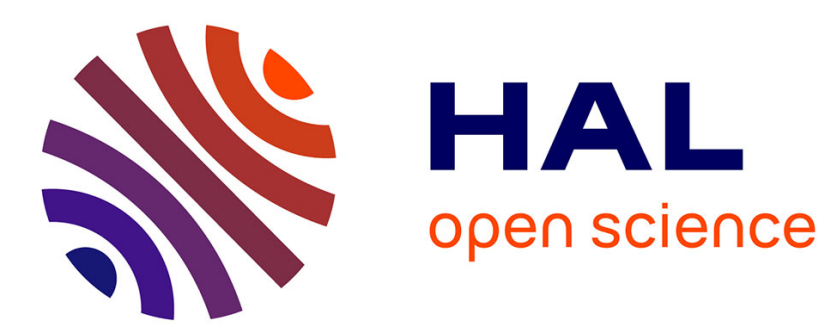

\title{
Modelling phase transition in metastable liquids: Application to cavitating and flashing flows.
}

\author{
Richard Saurel, Fabien Petitpas, Remi Abgrall
}

\section{To cite this version:}

Richard Saurel, Fabien Petitpas, Remi Abgrall. Modelling phase transition in metastable liquids: Application to cavitating and flashing flows.. Journal of Fluid Mechanics, 2008, 607, pp.313-350. inria-00333908

\section{HAL Id: inria-00333908 \\ https://hal.inria.fr/inria-00333908}

Submitted on 24 Oct 2008

HAL is a multi-disciplinary open access archive for the deposit and dissemination of scientific research documents, whether they are published or not. The documents may come from teaching and research institutions in France or abroad, or from public or private research centers.
L'archive ouverte pluridisciplinaire HAL, est destinée au dépôt et à la diffusion de documents scientifiques de niveau recherche, publiés ou non, émanant des établissements d'enseignement et de recherche français ou étrangers, des laboratoires publics ou privés. 


\title{
Modelling phase transition in metastable liquids. Application to cavitating and flashing flows
}

\author{
RICHARD SA UREL ${ }^{1}{ }^{3} \dagger$, F A B IEN PET I T P A S ${ }^{1}$ \\ AND REMIABGRALL $\mathbf{L}^{23}$ \\ ${ }^{1}$ Polytech'Marseille, Aix-Marseille Université and SMASH Project UMR CNRS 6595 - IUSTI - \\ INRIA, 5 rue E. Fermi, 13453 Marseille Cedex 13, France. \\ ${ }^{2}$ SCALAPPLIX Project. INRIA and Institut de Mathématiques, Université de Bordeaux I, \\ 351 cours de la Libération, 33405 Talence Cedex. France. \\ ${ }^{3}$ Also, University Institute of France, same addresses \\ (Received ?? and in revised form ??)

\begin{abstract}
A hyperbolic two-phase flow model involving five partial differential equations is built for liquid-gas interface modelling. The model is able to deal with interfaces of simple contact where normal velocity and pressure are continuous as well as transition fronts where heat and mass transfer occur, involving pressure and velocity jumps. These fronts correspond to extra waves into the system. The model involves two temperatures and entropies but a single pressure and a single velocity. The closure is achieved by two equations of state that reproduce the phase diagram when equilibrium is reached. Relaxation toward equilibrium is achieved by temperature and chemical potential relaxation terms whose kinetics is considered infinitely fast at specific locations only, typically at evaporation fronts. Doing so, metastable states are involved for locations far from these fronts. Computational results are compared to the experimental ones of Simoes-Moreira \& Shepherd (1999). Computed and measured front speeds are of the same order of magnitude and the same tendency of increasing front's speed versus initial temperature is reported. Moreover, the limit case of evaporation fronts propagating in highly metastable liquids with the Chapman-Jouguet speed is recovered as an expansion wave of the present model in the limit of stiff thermal and chemical relaxation.
\end{abstract}

\section{Introduction}

When a liquid initially in thermodynamic equilibrium undergoes strong rarefaction waves, it may reach a metastable state where the temperature is higher than the saturated one at the final pressure of the expanded state. Then the superheated liquid releases its metastable energy (stored as internal energy) very quickly, even explosively, producing either pure vapor, if the liquid is retrograde (Chaves (1984); Thompson et al. (1987); Kurschat et al. (1992)). or liquid-vapor mixture at high velocity. This phenomenon is often called cavitation. Such situations appear frequently in nature and in many industrial applications, as liquid flows around hypervelocity projectiles, submarine airfoils, or inside nozzles such as fuel injector systems. Cavitation in these systems always produces

$\dagger$ E-mail adress: richard.saurel@polytech.univ-mrs.fr 


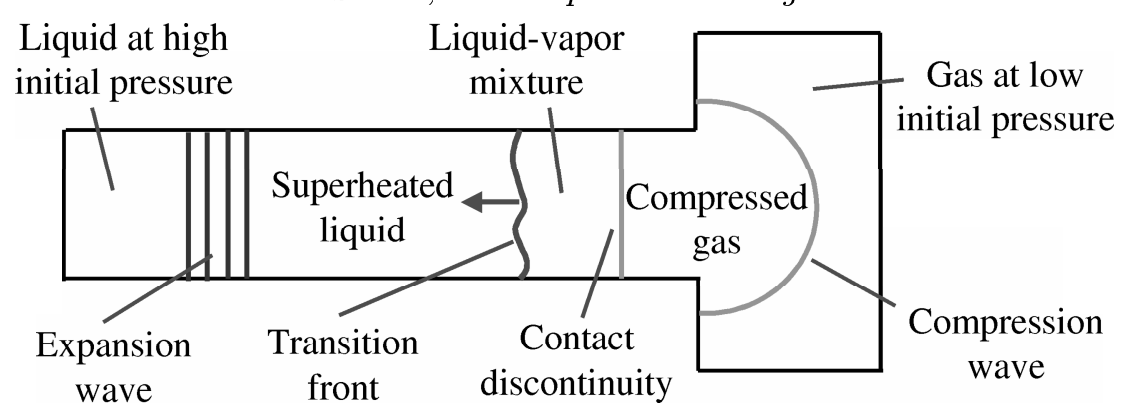

Figur. 1. Simplified experimental setup of the expansion tube used by Simoes-Moreira \& Shepherd (1999) and associated waves.

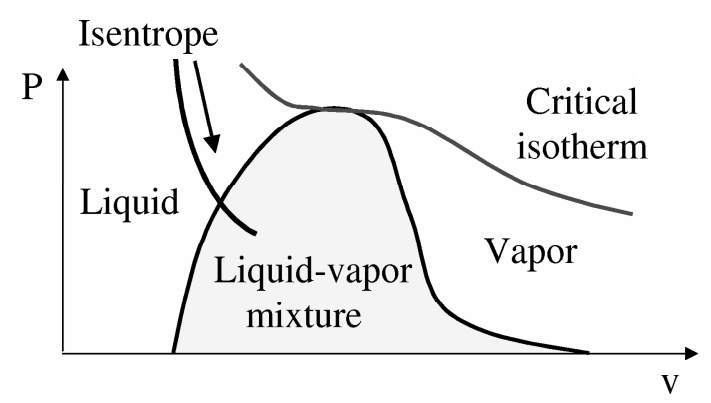

Figur. 2. Liquid thermodynamic path associated to the expansion wave producing a superheated liquid

strong disturbances. In most applications, cavitation appears as a multidimensional phenomenon due to geometrical effects. This multidimensional character complicates both experiments and theoretical approaches. By using one-dimensional expansion tubes several authors (Grolmes \& Fauske (1974); Chaves (1984); Thompson et al. (1987); Hill \&. Sturtevant (1990); Frost et al. (1991); Kurschat et al. (1992); Simoes-Moreira \& Shepherd (1999); Reinke \&. Yadigaroglu (2001)) succeeded in isolating the main phenomenon we propose to summarize hereafter. These experiments consisted in connecting a vertical tube filled with a liquid in thermodynamic equilibrium at atmospheric pressure (or higher) to a very low-pressure chamber (figure 1).

As soon as the membrane between the liquid and the vacuum chamber is ruptured, rarefaction waves propagate through the liquid producing a superheated liquid (figure 2).

Then a subsonic phase-transition front propagates through the superheated liquid producing a high velocity liquid-vapor mixtıre in thermodynamic equilibrium towards the low-pressure chamber. The front velocity is approximately $1 \mathrm{~m} / \mathrm{s}$ while the ejected mixture velocity is of the order of $100 \mathrm{~m} / \mathrm{s}$.

These experimental observations indicate that both liquid and vapor compressibilities have to be considered. They also report the presence of an acoustic wave (expansion wave) preceding the evaporation front, itself preceding a contact discontinuity and a compression wave as represented in the figure 1 . The corresponding waves pattern is shown in the $(x, t)$ diagram of figure 3 .

Expansion effects in industrial systems are often due to geometrical effects, as for example in nozzles where cavitation appears at locations where the pressure is very low and the liquid superheated (figure 4 ).

The aim of the present paper is to develop a model able to deal with metastable states and evaporation front dynamics, as well as the other waves shown in figure 3 . The model 


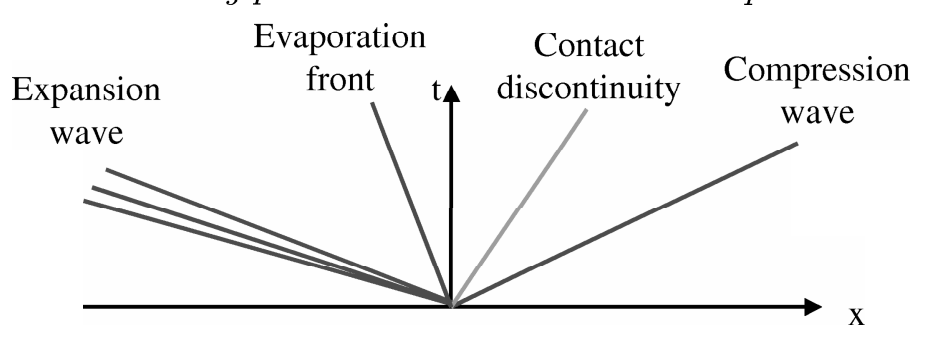

FiguRE 3. A typical waves pattern of cavitating systems

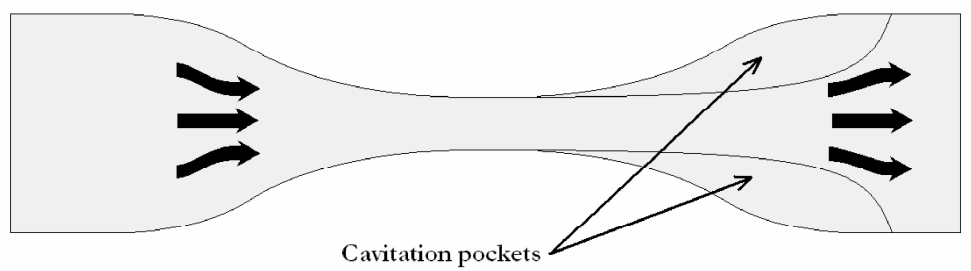

Figur.F 4. Cavitating flow in a nozzle. Cavitation pockets appear in the divergent of the nozzle where geometrical expansion produces metastable liquid state.

must also be able to deal with interfaces separating a liquid and a non condensable gas. Indeed, mass transfer occurs only under specific thermodynamic conditions that do not necessarily correspond to the local thermodynamic state at the interfaces. Thus the model must be able to deal with interfaces of simple contact as well as evaporating interfaces.

The paper is organized as follows. Existing model are reviewed and a critical analysis is given in $\S 2$. Justification of the choice for temperature non equilibrium model is done. In $\S 3$, starting from the total non equilibrium model of Baer \& Nunziato (1986) an asymptotic analysis is carried out in order to derive a single velocity and pressure two phase flow model including heat transfers. This model has the ability to solve interface. problems with non miscible fluids. Its thermodynamic closure is examined in $\$ 4$. Mass transfer modelling is addressed in $\$ 5$. Closure relations are obtained by examining the entropy production in each phase and in the mixture. The basic ingredients to solve the hyperbolic system with heat and mass transfer are reported in $\$ 6$. Numerical results are reported in $\$ 7$. The limit case of interfaces separating pure non miscible fluids is examined. Then mass transfer is introduced producing extra waves into the system. Computed results are compared to the experimental ones of Simoes-Moreira \& Shepherd (1999). The Chapmann-Jouguet (CJ) kinetic relation proposed by Chaves (1984) for evaporation fronts propagation in highly metastable liquids is recovered and justified as an eigenvalue of the limit system with stiff thermal and chemical relaxation.

\section{Review of existing models}

Essentially two classes of models are available in the literature. The first class corresponds to pressure and temperature equilibrium models, the second class being related to temperature non-equilibrium models.

\subsection{Pressure and temperature equilibrium models}

Four models at least belong to this category, with increasing level of complexity: 
- The mixtıre Euler equations with a cubic equation of state (EOS). A prototype of such EOS is the van der Waals one. With such models a loss of hyperbolic occurs in the spinodal region. In other words, the square sound speed may becomes negative and waves propagation has no physical sense (Menikoff \& Plohr (1989)).

- The mixtıre Euler equations can also be used with a tabular EOS or a combination of pure phase EOSs with assumption of pressure, temperature and chemical potential equilibrium in the two-phase region (see for example Saurel et. al. 1999; Lion \& Edwards 1999). Such model removes the preceding loss of hyperbolicity. However, the mixture is assumed to evolve under thermodynamic equilibrium. thus omitting metastable states. Also, the model is unable to deal with material interfaces separating a liquid and a non-condensable gas. Hence, it has a restricted domain of application.

- The mixture Euler equations are sometimes augmented by a mass fraction equation with a relaxation term (Faucher et al. (2000)), as frequently done for mixtures of reacting gases. This four equations model is unable to solve interfaces between a liquid and a non-condensable gas (water/air for example). This is due to its isothermal closure not compatible with interface conditions (equal pressures and normal velocities but not temperatures). Moreover, determination of the mass relaxation term is an issue.

- Models derived from the second-gradient theory (Cahn \& Hilliard (1958)). In this approach, the interface is described as a diffuse zone with a capillary length scale that has to be resolved. This results in severe restrictions as this zone length is typically of the order of one nano-meter. To do practical simulations, at the scale on an individual bubble for example, the interface has to be thickened (Jamet et al. (2001)). Such procedure has important consequences on the model ability to deal with metastable states. Also, interfaces with large density gradients are very difficult to solve, as well as interfaces separating immiscible fluids (water/air for example).

\subsection{Temperature non-equilibrium models}

Three models at least belong to this category:

- The most conventional temperature non-equilibrium model is the 6 equations model obtained for example with averaging methods (Ishii (1975); Delhaye \& Boure (1982)). It involves a balance equation of mass, momentum and energy per fluid. Contrarily to the preceeding models, the mixture evolves with two velocities. It is present in the literature with two options:

i) One of the phases is considered incompressible, the mixture pressure being those of the gas phase. Such option is not compatible with the presence of rarefaction waves in the liquid phase, whose presence is mandatory in cavitating flows.

ii) Both phases are compressible and the closure is achieved with the pressure equilibrium assumption (Butler et, al. (1982)).

The 6 equation model, with both options has a restricted domain of hyperbolicity. This results in a restricted validity to problems where transient wave propagation is not essential. Moreover, it is unable to solve interface problems (water/air for example).

- The 7 equations model (Baer \& Nunziato (1986)) (and its variants) is unconditionally hyperbolic and is able to deal with a wide range of applications. It is composed of the same 6 equations as previously, augmented by an evolution equation for the volume fraction of one of the constituents. Its ability to solve interface problems as well as fluid mixtures with several velocities was demonstrated in Saurel \& Abgrall (1999), Saurel \&. Le Metayer (2001) and Saurel et al. (2003). This model has been extended to the propagation of evaporation fronts in cavitating systems (Le Metayer et al. (2005)). The four waves present in the figure 3 are correctly captured and the model is able to deal with metastable states. It has been used for practical computations and validated against 
experiments of supercavitation. However it is quite complex to code and does not provide information about the structure of the evaporation front. as the front is considered as a sharp discontinuity.

- The last model involves a single pressure and velocity. It is composed of two mass balance equations, one mixture momentum equation, one mixture energy equation and a volume fraction equation. This 5 equations model (Kapila et al. (2001)) is unconditionally hyperbolic. Its ability to solve interface problems with compressible fluids was demonstrated in Murrone \& Guillard (2004), Perigaud \&. Saurel (2005) and Petitpas et al. (2007). It was extended in Perigaud \& Saurel (2005) to capillary and viscous effects. Contrarily to the Cahn \&. Hilliard (1958) model, the interface has no capillary length scale. This has important consequences on numerical resolution as well as its ability to deal with large density ratios. Another important difference is also present regarding thermodynamic closure. Two pure phase EOSs are used instead of a single one, like van der Waals one. The model remains out of thermal equilibrium and the presence of temperature and chemical potential differences will be used hereafter to derive a relaxation model able to deal with evaporation fronts.

This last model is the starting point of the present analysis. Its derivation in the presence of heat and mass transfer is detailed hereafter. Its thermodynamic closure, based on two equations of state is detailed. The building of kinetic terms that make the system relax toward equilibrium is done on the basis of the entropy inequality. With this kinetic: closure and special treatment of stiff relaxation, the model is shown to be able to compute evaporation fronts by solving their internal structure. Metastable states are involved, as well as shock, rarefaction and interface dynamics. Dynamic appearance of cavitation pockets is studied in the following limit situations:

- simple mechanical pocket growth.

- mechanical pocket growth with heat and mass exchanges.

\section{The flow model when there is no mass transfer}

The present approach is based on a five equations model with a single pressure and a single velocity but two temperatures and entropies. Mass transfers will be modeled as relaxation effects. Before doing such extension, the flow model in absence of mass transfer is derived. It is obtained in the asymptotic limit of a non equilibrium two-phase, two pressures and two velocities hyperbolic multiphase flow model. The following asymptotic reduction follows the lines of Kapila et al. (2001).

\subsection{The "parent" model}

The starting point of the present analysis is the Baer \& Nunziato (1986) with symmetric closure relations (Saurel et al. 2003: Chinnayya et al. 2004): 


$$
\left\{\begin{array}{l}
\frac{\partial \alpha_{1}}{\partial t}+\boldsymbol{u}_{I} \cdot \nabla \alpha_{1}=\mu\left(p_{1}-p_{2}\right) \\
\frac{\partial \alpha_{1} \rho_{1}}{\partial t}+\operatorname{div}\left(\alpha_{1} \rho_{1} \boldsymbol{u}_{1}\right)=0 \\
\frac{\partial \alpha_{1} \rho_{1} \boldsymbol{u}_{1}}{\partial t}+\operatorname{div}\left(\alpha_{1} \rho_{k} \boldsymbol{u}_{k} \otimes \boldsymbol{u}_{k}\right)+\nabla\left(\alpha_{1} p_{1}\right)=p_{I} \boldsymbol{\nabla} \alpha_{1}+\lambda\left(\boldsymbol{u}_{2}-\boldsymbol{u}_{1}\right) \\
\frac{\partial \alpha_{1} \rho_{1} E_{1}}{\partial t}+\operatorname{div}\left(\alpha_{1}\left(\rho_{1} E_{1}+p_{1}\right) \boldsymbol{u}_{1}\right) \\
=p_{I} \boldsymbol{u}_{I} \cdot \boldsymbol{\nabla} \alpha_{1}+\lambda \boldsymbol{u}_{I} \cdot\left(\boldsymbol{u}_{2}-\boldsymbol{u}_{1}\right)-p_{I} \mu\left(p_{1}-p_{2}\right)+Q_{1} \\
\frac{\partial \alpha_{2} \rho_{2}}{\partial t}+\operatorname{div}\left(\alpha_{2} \rho_{2} \boldsymbol{u}_{2}\right)=0, \\
\frac{\partial \alpha_{2} \rho_{2} \boldsymbol{u}_{2}}{\partial t}+\operatorname{div}\left(\alpha_{2} \rho_{k} \boldsymbol{u}_{k} \otimes \boldsymbol{u}_{k}\right)+\nabla\left(\alpha_{2} p_{2}\right)=p_{I} \boldsymbol{\nabla} \alpha_{2}-\lambda\left(\boldsymbol{u}_{2}-\boldsymbol{u}_{1}\right) \\
\frac{\partial \alpha_{2} \rho_{2} E_{2}}{\partial t}+\operatorname{div}\left(\alpha_{2}\left(\rho_{2} E_{2}+p_{2}\right) \boldsymbol{u}_{2}\right) \\
\quad=p_{I} \boldsymbol{u}_{I} \cdot \boldsymbol{\nabla} \alpha_{2}-\lambda \boldsymbol{u}_{I} \cdot\left(\boldsymbol{u}_{2}-\boldsymbol{u}_{1}\right)+p_{I} \mu\left(p_{1}-p_{2}\right)-Q_{1}
\end{array}\right.
$$

We denote respectively by $\alpha_{k}, \rho_{k}, \boldsymbol{u}_{k}, p_{k}, E_{k}$ and $e_{k}$ the volume fraction, the density, the velocity vector, the pressure, the total specific energy and the internal specific energy of the phase $\mathrm{k}$. The total specific energy is defined by $E_{k}=e_{k}+\boldsymbol{u}_{k}^{2} / 2$.

The heat transfer term $Q_{1}$ is simply modeled by $Q_{1}=H\left(T_{2}-T_{1}\right)$ where $H=h . S_{I}$ involves the convective heat transfer coefficient $h$ and the specific exchange surface $S_{I}$. This system guarantees conservation for the mixture and is frame invariant.

The interaction terms that appear in the right hand side express the effects which drive the system to mechanical equilibrium by the way of relaxation coefficients. Following Saurel et al. (2003) where the continuous limit of the discrete two-phase flow equations derived in Abgrall \& Saurel (2003) is obtained, symmetric closure relations are available:

$$
\mu=\frac{S_{I}}{Z_{1}+Z_{2}} \text { and } \lambda=Z_{1} Z_{2} \mu
$$

where $Z$ represents the acoustic impedance $(Z=\rho c)$. The average interfacial pressure and velocity are given by:

$$
\begin{aligned}
& p_{I}=\frac{Z_{1} p_{2}+Z_{2} p_{1}}{Z_{1}+Z_{2}}+\operatorname{sign}\left(\frac{\partial \alpha_{1}}{\partial x}\right) \frac{\left(u_{2}-u_{1}\right) Z_{1} Z_{2}}{Z_{1}+Z_{2}}, \\
& u_{I}=\frac{Z_{1} u_{1}+Z_{2} u_{2}}{Z_{1}+Z_{2}}+\operatorname{sign}\left(\frac{\partial \alpha_{1}}{\partial x}\right) \frac{p_{2}-p_{1}}{Z_{1}+Z_{2}} .
\end{aligned}
$$

The system (3.1) with this choice of interface variables and relaxation coefficient is consistent with the second law of thermodynamics. Other estimates are also possible, one other choice is given by Baer \&. Nunziato (1986):

$$
\begin{aligned}
& p_{I}=p_{1}, \\
& u_{I}=u_{2} .
\end{aligned}
$$

In the present study, where stiff mechanical relaxation is considered, the estimates (3.2) and (3.3) can be used indifferently. However, in the general case of finite relaxation 
rate, the estimates (3.2) are able to enforce interface conditions at each volume fraction discontinuity (equality of normal velocities and pressure) automatically (Abgrall \& Saurel 2003). This system is unconditionally hyperbolic and admits the characteristic waves speeds: $u_{k}, u_{k}+c_{k}, u_{k}-c_{k}$ for each phase $\mathrm{k}$ and the interface velocity $u_{I}$.

For the present application, this system involves unnecessary effects (two velocities and two pressures) and a reduced model is preferred. It is indeed important to determine the simplest model involving the pertinent physics. The reduction of system (3.1) is done in the following subsection.

\subsection{Reduced model}

The two-phase flow model (3.1) can be written using primitive variables, namely

$$
\frac{\partial U}{\partial t}=F(U)+\Phi(U),
$$

with

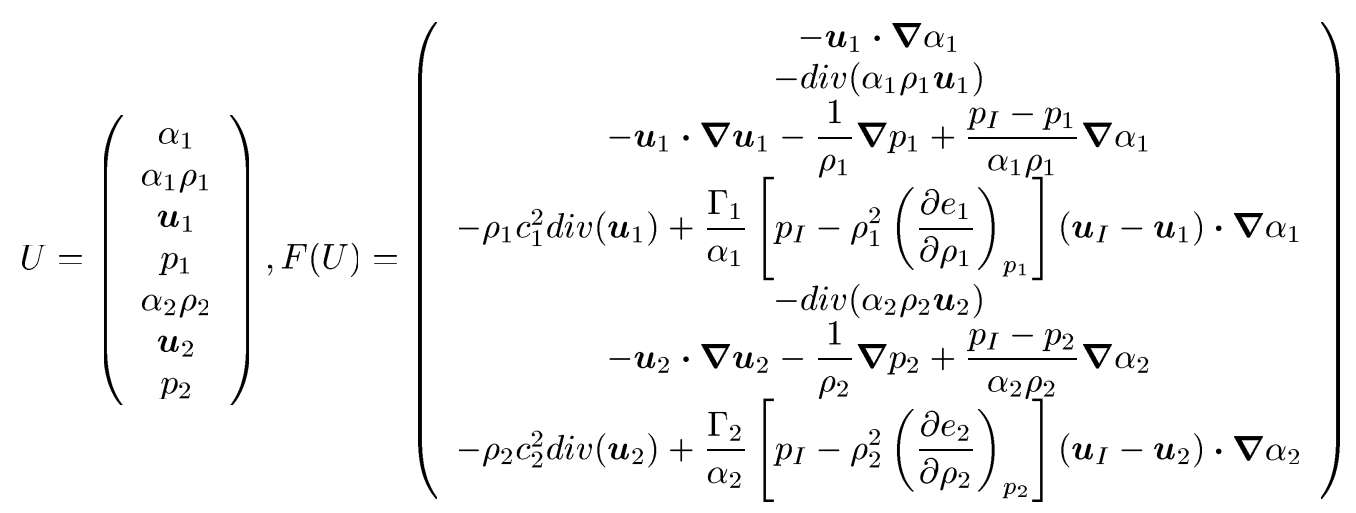

and

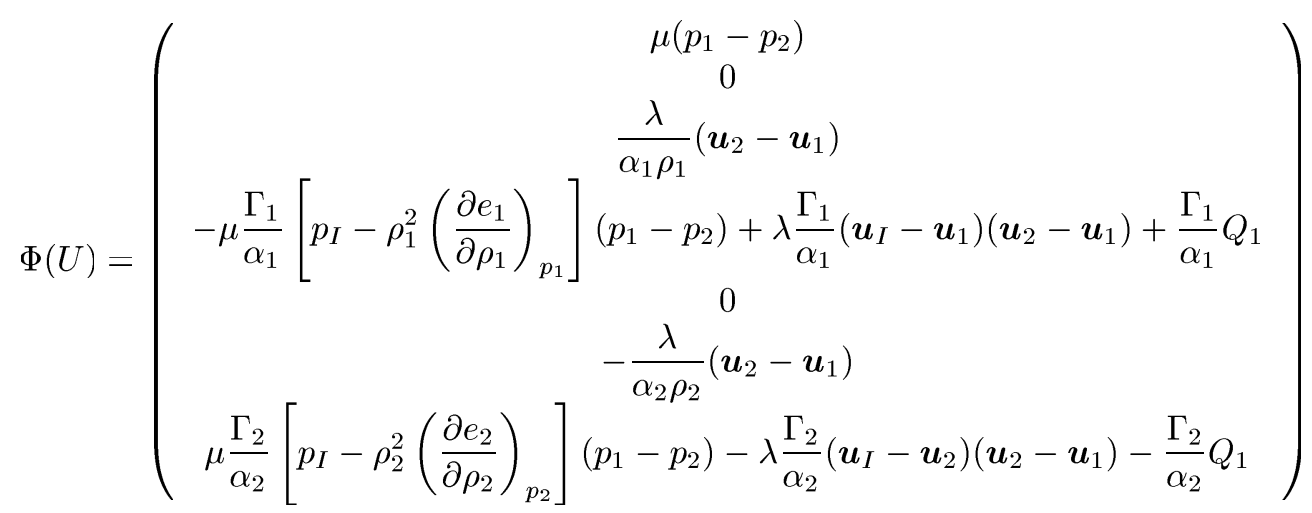

Here, $c_{k}$ represents the speed of sound of phase $\mathrm{k}$ :

$$
\forall k=1,2 \quad c_{k}^{2}=\frac{\frac{p_{k}}{\rho_{k}^{2}}-\left(\frac{\partial e_{k}}{\partial \rho_{k}}\right)_{p_{k}}}{\left(\frac{\partial e_{k}}{\partial p_{k}}\right)_{\rho_{k}}} .
$$

and $\Gamma_{k}$ represents the Gruneisen coefficient of phase $k$ :

$$
\forall k=1,2 \quad \Gamma_{k}=v_{k}\left(\frac{\partial p_{k}}{\partial e_{k}}\right)_{\rho_{k}}
$$


where $v_{k}=1 / \rho_{k}$.

The reduced system is obtained in the limit of stiff mechanical relaxation:

$$
\mu=\frac{1}{\epsilon}, \lambda=\frac{1}{\epsilon} \quad \text { where } \epsilon \rightarrow 0^{+} .
$$

Note that infinite relaxation parameters are consistent with the expression of pressure and velocity relaxation parameters linked by the relation $\lambda=Z_{1} Z_{2} \mu$. It is also important to note that heat transfer effects are not considered in the same limit, as we are seeking a temperature non equilibrium model.

With these notations, the preceding system becomes:

$$
\frac{\partial U}{\partial t}=F(U)+\frac{1}{\epsilon} \Psi(U)+Q(U)
$$

with:

$$
\Psi(U)=\left(\begin{array}{c}
\left(p_{1}-p_{2}\right) \\
0 \\
\frac{Z_{1} Z_{2}}{\alpha_{1} \rho_{1}}\left(\boldsymbol{u}_{2}-\boldsymbol{u}_{1}\right) \\
-\frac{\Gamma_{1}}{\alpha_{1}} K_{1}\left(p_{1}-p_{2}\right)+\frac{Z_{1} Z_{2} \Gamma_{1}}{\alpha_{1}}\left(\boldsymbol{u}_{I}-\boldsymbol{u}_{1}\right)\left(\boldsymbol{u}_{2}-\boldsymbol{u}_{1}\right) \\
0 \\
-\frac{Z_{1} Z_{2}}{\alpha_{2} \rho_{2}}\left(\boldsymbol{u}_{2}-\boldsymbol{u}_{1}\right) \\
\frac{\Gamma_{2}}{\alpha_{2}} K_{2}\left(p_{1}-p_{2}\right)-\frac{Z_{1} Z_{2} \Gamma_{2}}{\alpha_{2}}\left(\boldsymbol{u}_{I}-\boldsymbol{u}_{2}\right)\left(\boldsymbol{u}_{2}-\boldsymbol{u}_{1}\right)
\end{array}\right), Q(U)=\left(\begin{array}{c}
0 \\
0 \\
0 \\
\frac{\Gamma_{1}}{\alpha_{1}} Q_{1} \\
0 \\
0 \\
-\frac{\Gamma_{2}}{\alpha_{2}} Q_{1}
\end{array}\right)
$$

Here, $F$ and $\Psi$ are regular enough functions evaluated at a state $U$ which is close to the mechanical equilibrium state $U_{0}$. We can set

$$
U^{\epsilon}=U_{0}+\epsilon U_{1}+O\left(\epsilon^{2}\right)
$$

and the system (3.4) becomes:

$$
\begin{aligned}
\frac{\partial U_{0}}{\partial t}+\epsilon \frac{\partial U_{1}}{\partial t}=F\left(U_{0}\right) & +\epsilon \frac{\partial F(U)}{\partial U}\left(U_{0}\right) U_{1}+\frac{1}{\epsilon} \Psi\left(U_{0}\right)+\frac{\partial \Psi(U)}{\partial U}\left(U_{0}\right) U_{1} \\
& +Q\left(U_{0}\right)+\epsilon \frac{\partial Q(U)}{\partial U}\left(U_{0}\right) U_{1}+O\left(\epsilon^{2}\right) .
\end{aligned}
$$

Since $U_{0}$ is an equilibrium state,

$$
\Psi\left(U_{0}\right)=0
$$

and, the system under reduced form becomes:

$$
\frac{\partial U_{0}}{\partial t}=F\left(U_{0}\right)+\frac{\partial \Psi(U)}{\partial U}\left(U_{0}\right) U_{1}+Q\left(U_{0}\right)
$$

Condition (3.5) implies that in the asymptotic state, the system has to verify:

$$
\boldsymbol{u}_{1}^{0}=\boldsymbol{u}_{2}^{0}=\boldsymbol{u}^{0}
$$


and

$$
p_{1}^{0}=p_{2}^{0}=p^{0}
$$

These constraints used in (3.6) give the reduced model that takes into account heat transfer effects:

$$
\left\{\begin{array}{l}
\frac{\partial \alpha_{1}}{\partial t}+\boldsymbol{u} \cdot \nabla \alpha_{1}=K\left(\alpha_{1}, \rho_{1}, \rho_{2}, p\right) \operatorname{div}(\boldsymbol{u})+\frac{\alpha_{1} \alpha_{2}}{\alpha_{2} \rho_{1} c_{1}^{2}+\alpha_{1} \rho_{2} c_{2}^{2}}\left(\frac{\Gamma_{1}}{\alpha_{1}}+\frac{\Gamma_{2}}{\alpha_{2}}\right) Q_{1} \\
\frac{\partial \alpha_{1} \rho_{1}}{\partial t}+\operatorname{div}\left(\alpha_{1} \rho_{1} \boldsymbol{u}\right)=0 \\
\frac{\partial \alpha_{2} \rho_{2}}{\partial t}+\operatorname{div}\left(\alpha_{2} \rho_{2} \boldsymbol{u}\right)=0 \\
\frac{\partial \rho \boldsymbol{u}}{\partial t}+\operatorname{div}(\rho \boldsymbol{u} \otimes \boldsymbol{u})+\nabla p=0 \\
\frac{\partial \rho E}{\partial t}+\operatorname{div}(\boldsymbol{u}(\rho E+p))=0
\end{array}\right.
$$

where $K=\alpha_{1} \alpha_{2}\left(\rho_{2} c_{2}^{2}-\rho_{1} c_{1}^{2}\right) /\left(\alpha_{2} \rho_{1} c_{1}^{2}+\alpha_{1} \rho_{2} c_{2}^{2}\right)$. The upperscript " 0 " has been omitted for the sake of clarity. The mixture variables are defined by: $\rho=\alpha_{1} \rho_{1}+\alpha_{2} \rho_{2}$ and $E=Y_{1} E_{1}+Y_{2} E_{2}$. where $Y_{k}=\alpha_{k} \rho_{k} / \rho$ denotes the mass fraction of the phase $k$.

\section{Thermodynamic closure}

In order to circumvent the difficulty of models having negative square speed of sound in the two-phase region, the present model uses two equations of state (EOS). Each fluid possesses its own EOS. In the present paper: we consider "stiffened gas" (SG) equations of state. but the method can be easily generalised to more complex convex equations of state. Our goal in using this type of EOS is to handle the essential of the physics and thermodynamics under simple analytical formula. Moreover this E()S is the simplest prototype that contains the main physical properties of pure fluids: attractive and repulsive molecular effects. Doing so, each fluid has its own thermodynamics and in particular its own entropy. In the present modelling of mass transfer, detailed in the next section, relaxation towards equilibrium is achieved by a kinetic process, contrarily to van der Waals modelling where mass transfer is a thermodynamic path. It is the reason why the present modelling preserves hyperbolicity during mass transfer.

However, when equilibrium is reached, conventional properties of the phase diagram have to be recovered (latent heat of vaporization. saturation temperature) that depend on pressure or temperature. In other words, the two pure fluids EOS must be connected by some constraints. These constraints are used for the determination of the various constants involved in these EOSs. 
For each phase the thermodynamic state is determined by the SG EOS that reads:

$$
\begin{aligned}
e(p, v) & =\frac{p+\gamma p_{\infty}}{(\gamma-1)} v+q \\
v(p, T) & =\frac{(\gamma-1) C_{v} T}{p+p_{\infty}} \\
h(T) & =\gamma C_{v} T+q \\
g(p, T) & =\left(\gamma C_{v}-q^{\prime}\right) T-C_{v} T \log \frac{T^{\gamma}}{\left(p+p_{\infty}\right)^{(\gamma-1)}}+q
\end{aligned}
$$

Where $e, v=1 / \rho, p, T, h$ and $g$ are respectively the internal energy, the specific volume: the pressure, the temperature, the enthalpy and the Gibbs free energy of the considered phase. The constants, characteristic of each fluid are: $\gamma, p_{\infty}, C_{v}, q$ and $q^{\prime}$.

A method to determine these parameters in gas-liquid systems is given in Le Metayer et. al. (2004). The coupling of gas and liquid parameters is particularly examined. This method is summarized hereafter.

\subsection{Method to determine SG EOS parameters}

Parameters of the SG EOS are determined from experimental curves for each fluid. In the case we are interested in (liquid in presence of its vapor), we need the saturation curves. The experimental data needed are : $p=p_{s a t}(T), h_{l, e x p}(T), h_{g, \exp }(T), v_{l, \exp }(T)$, $v_{g, \exp }(T)$ and the latent heat of vaporization $L_{v, \exp }(T)=h_{g, e x p}(T)-h_{l, e x p}(T)$.

(a) From the expression of enthalpy $(4.1 c)$, we have:

$$
\forall k=l, g \quad \frac{d h_{k}}{d T}=\gamma_{k} C_{v, k}=C_{p, k} .
$$

This permits to determine the average heat capacity coefficients $C_{p, k}$ by a linear approximation between two reference states () and 1 as:

$$
\forall k=l, g \quad C_{p, k}=\frac{h_{k, e x p}\left(T_{1}\right)-h_{k, e x p}\left(T_{0}\right)}{T_{1}-T_{0}} .
$$

This also allows the calculation of the reference energies:

$$
\forall k=l, g \quad q_{k}=h_{k, \exp }\left(T_{0}\right)-C_{p, k} T_{0} .
$$

(b) Then, by using the experimental curve $p=p_{\text {sat }}(T)$, the specific volume is expressed as:

$$
\forall k=l, g \quad v_{k}(T)=\frac{\left(C_{p, k}-C_{v, k}\right) T}{p_{s a t}(T)+p_{\infty, k}} .
$$

Under logarithmic differentiation, we have:

$$
\forall k=l, g \quad d \log \left(v_{k}(T)\right)=d \log (T)-d \log \left(p_{\text {sat }}(T)+p_{\infty, k}\right) .
$$

The integration of this equation between the two reference states () and 1 yields:

$$
\begin{aligned}
\forall k=l, g \quad \log \left(v_{k}\left(T_{1}\right)\right) & -\log \left(v_{k}\left(T_{0}\right)\right) \\
& =\log \left(T_{1}\right)-\ln \left(T_{0}\right)-\log \left(p_{\text {sat }}\left(T_{1}\right)+p_{\infty, k}\right)+\log \left(p_{\text {sat }}\left(T_{0}\right)+p_{\infty, k}\right)
\end{aligned}
$$

These expression allows the calculation of the coefficients $p_{\infty, k}$ :

$$
\forall k=l, g \quad p_{\infty, k}=\frac{v_{k}\left(T_{0}\right) T_{1} p_{s a t}\left(T_{0}\right)-v_{k}\left(T_{1}\right) T_{0} p_{s a t}\left(T_{1}\right)}{v_{k}\left(T_{1}\right) T_{0}-v_{k}\left(T_{0}\right) T_{1}}
$$


(c) Then (4.2) applied to the reference state 0 provides the approximation of $C_{v, k}$ :

$$
\forall k=l, g \quad C_{v, k}=C_{p, k}-\frac{v_{k}\left(T_{0}\right)}{T_{0}}\left(p_{s a t}\left(T_{0}\right)+p_{\infty, k}\right) .
$$

(d) The approximation for $\gamma_{k}$ follows :

$$
\forall k=l, g \quad \gamma_{k}=\frac{C_{p, k}}{C_{v, k}}
$$

(e) At thermodynamic equilibrium, the two Gibbs free energy have to be equal ( $g_{g}=$ $g_{l}$ ) with the definition $\left.(4.1 d)\right)$. This implies:

$$
\log \left(p+p_{\infty, v}\right)=A+\frac{B}{T}+C \log (T)+D \log \left(p+p_{\infty, l}\right)
$$

where A, B, C and I) depends on the SG EOS parameters:

$$
A=\frac{C_{p, l}-C_{p, g}+q_{g}^{\prime}-q_{l}^{\prime}}{C_{p, g}-C_{v, g}}, B=\frac{q_{l}-q_{g}}{C_{p, g}-C_{v, g}}, C=\frac{C_{p, g}-C_{p, l}}{C_{p, g}-C_{v, g}}, \text { and } D=\frac{C_{p, l}-C_{v, l}}{C_{p, g}-C_{v, g}}
$$

The relation (4.3) is non linear but permits the computation of the theoretical curve $p=p_{\text {sat }}(T)$. Such computation needs the two entropy constants $q^{\prime}$. By convention, we take $q_{l}^{\prime}=0 \mathrm{~J} \cdot \mathrm{kg}^{-1} \cdot K^{-1}$ and choose $q_{g}^{\prime}$ in order to have the best fit between theoretical and experimental c:urves.

This algorithm for the determination of SG EOS parameters is very accurate provided that experimental curves saturation curves are quasi-linear. It means that the two reference states have to be sufficiently close. Near the critical point, restrictions appear. But far from this point, wide ranges of temperatures and pressures can be covered as shown hereafter.

\subsection{Results for water and dodecane}

As an illustration, results concerning liquid and vapor water and liquid and vapor dode(ane are presented.

For water, the chosen temperature range is $298-473 \mathrm{~K}$. The corresponding experimental data (Oldenbourg 1989) are :

$p_{\text {sat }}\left(T_{0}\right)=3166 \mathrm{~Pa}, \quad h_{l, \exp }\left(T_{0}\right)=104.7 \times 10^{3} \mathrm{~J} / \mathrm{Kg}, \quad h_{g, \exp }\left(T_{0}\right)=2473.4 \times 10^{3} \mathrm{~J} / \mathrm{Kg}$. $v_{g, \exp }\left(T_{0}\right)=42.4 m^{3} / K g$,

$p_{\text {sat }}\left(T_{1}\right)=15.510^{5} \mathrm{~Pa}, \quad h_{l, e x p}\left(T_{1}\right)=851.6 \times 10^{3} \mathrm{~J} / \mathrm{Kg}, \quad h_{g, \text { exp }}\left(T_{1}\right)=2733.7 \times 10^{3} \mathrm{~J} / \mathrm{Kg}$, $v_{g, \exp }\left(T_{1}\right)=0.124 m^{3} / K g$.

The results of the preceding algorithm are summarized in table 1 and the comparison between experimental and SG approximation curves is shown in figure 5. Obviously, some errors are present, essentially regarding the liquid specific volume where $25 \%$ maximum error is noticed. The reason is that the SG EOS is the simplest prototype involving repulsive and attractive potentials for condensed materials. With this particularly simple formulation it is possible to fit quite correctly saturation curves and phase diagram. If better accuracy is required, a more sophisticated model can be used (Mie Gruneisen EOS for example), to the price of a heavier method to determine the various constants. Our aim is not to build EOSs in this paper but to propose a general technique. The problem of defining a proper equation of state has been considered in Le Metayer et al. (2004) and the method we propose in this paper can be applied to more general EOS without any special conceptual difficulty. Once more, our aim is more to show that the new multiphase model, under simplified thermodynamic closure, is able to deal with evaporation waves and metastable states with reasonable accuracy. 


$\begin{array}{ccccccc} & p_{\infty, k}(\mathrm{~Pa}) & C_{p}(\mathrm{~J} / \mathrm{kg} . \mathrm{K}) & C_{v}(\mathrm{~J} / \mathrm{kg} . \mathrm{K}) & \gamma & q(\mathrm{~J} / \mathrm{kg}) & q^{\prime}(\mathrm{J} / \mathrm{kg} . \mathrm{K}) \\ \text { liquid } & 10^{9} & 4267 & 1816 & 2.35 & -1167 \times 10^{3} & 0 \\ \text { vapor } & 0 & 1487 & 1040 & 1.43 & 2030 \times 10^{3} & -23 \times 10^{3}\end{array}$

TABLE 1. SG EOS parameters for liquid and vapor water
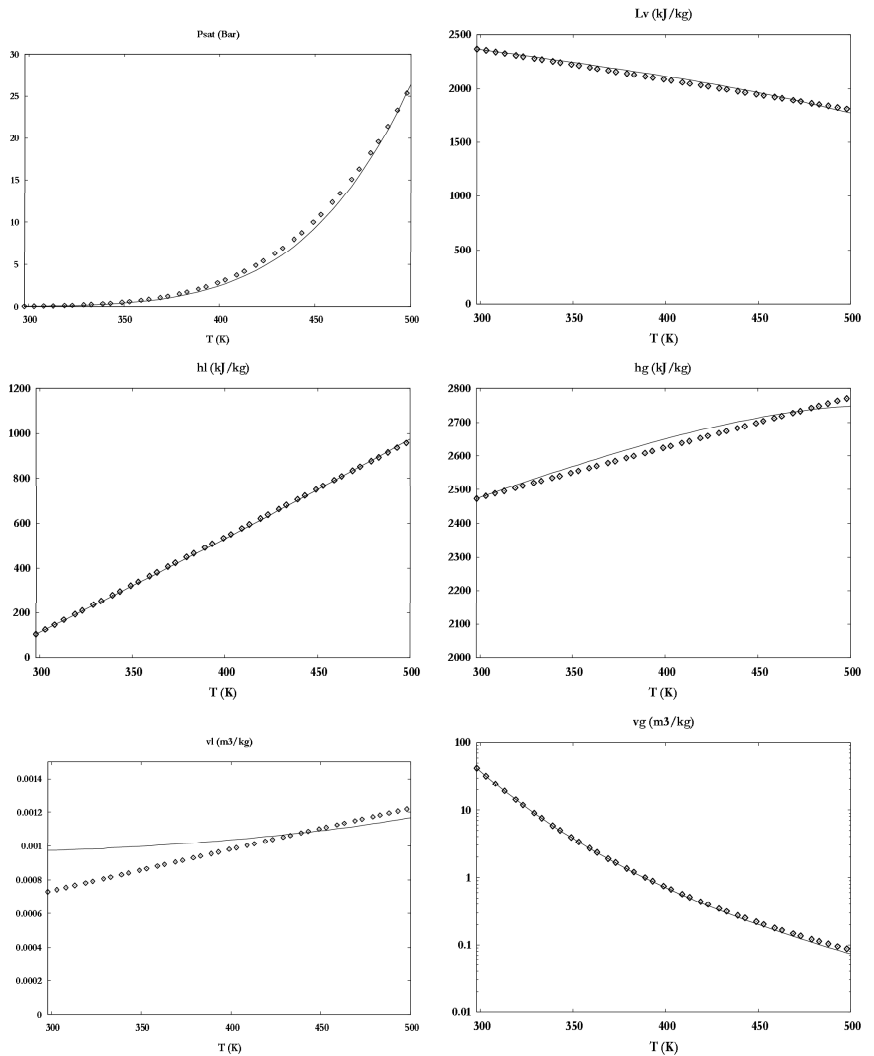

FiguR. 5. Saturation curves for water in the temperature range 298-473 K. Experimental curves are shown with lines and stiffened gas approximation are shown with symbols.

$\begin{array}{ccccccc} & p_{\infty, k}(\mathrm{~Pa}) & C_{p}(\mathrm{~J} / \mathrm{kg} . \mathrm{K}) & C_{v}(\mathrm{~J} / \mathrm{kg} . \mathrm{K}) & \gamma & q(\mathrm{~J} / \mathrm{kg}) & q^{\prime}(\mathrm{J} / \mathrm{kg} . \mathrm{K}) \\ \text { liquid } & 4 \times 10^{8} & 2534 & 1077 & 2.19 & -755 \times 10^{3} & 0 \\ \text { vapor } & 0 & 2005 & 1956 & 1.025 & -237 \times 10^{3} & -24 \times 10^{3}\end{array}$

TABLE 2. SG EOS parameters for liquid dodecane and its vapor

The same algorithm is used to determine thermodynamic parameters of dodecane. in the temperature range $298-473 K$. Phase diagram and associated data are available in Simoes-Moreira (1994). The corresponding SG E()S parameters are summarized in table 2. The same accuracy when compared with experimental data was observed. 


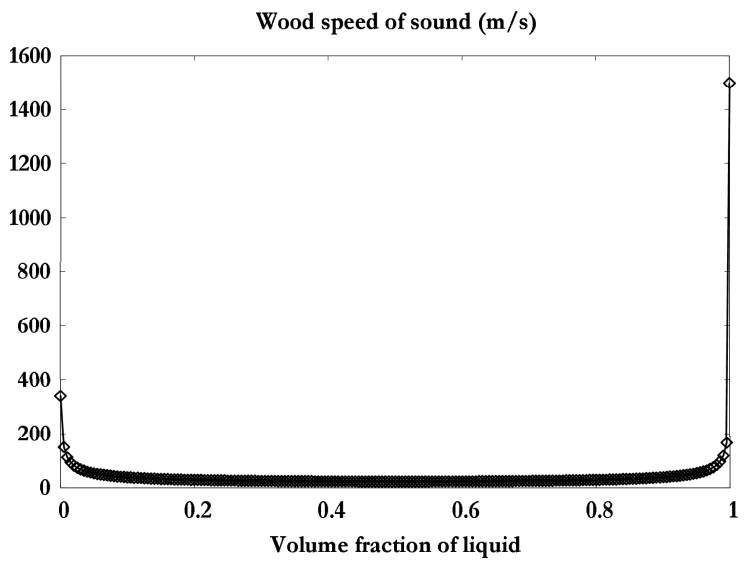

Figure 6. Wood's speed of sound versus liquid volume fraction for a liquid-vapor water mixtıre.

\subsection{Mixture $S G E() S$}

With the help of the phases EOS, the mixture EOS is easily obtained. The mixture specific internal energy definition writes:

$$
\rho e=\alpha_{1} \rho_{1} e_{1}+\alpha_{2} \rho_{2} e_{2}
$$

By using SG E()S (4.1a), each product $\rho_{k} e_{k}$ can be written as:

$$
\rho_{k} e_{k}=\frac{p_{k}+\gamma_{k} p_{\infty, k}}{\gamma_{k}-1}+\rho_{k} q_{k}
$$

Under pressure equilibrium. we obtain the closure relation for system (3.7):

$$
p\left(\rho, e, \alpha_{1}, \alpha_{2}, Y_{1}, Y_{2}\right)=\frac{\rho\left(e-Y_{1} q_{1}-Y_{2} q_{2}\right)-\left(\frac{\alpha_{1} \gamma_{1} p_{\infty, 1}}{\gamma_{1}-1}+\frac{\alpha_{2} \gamma_{2} p_{\infty, 2}}{\gamma_{2}-1}\right)}{\frac{\alpha_{1}}{\gamma_{1}-1}+\frac{\alpha_{2}}{\gamma_{2}-1}}
$$

With this mixture EOS, the flow model (3.7) reproduces propagation of acoustic disturbance at the Wood speed of sound (Wood 1930):

$$
\frac{1}{\rho c_{u i}^{2}}=\frac{\alpha_{1}}{\rho_{1} c_{1}^{2}}+\frac{\alpha_{2}}{\rho_{2} c_{2}^{2}}
$$

This sound speed has a non-monotonic behavior versus volume fraction, as shown in figure 6 . The system (3.7) is strictly hyperbolic with the characteristic waves speeds: $u+c_{w}: u-c_{w}$ and $u$.

\section{Mass transfer modelling}

\subsection{Basic ideas}

As mentioned in Section 2, it is well known (Menikoff \& Plohr 1989) that van der Waals or cubic EOS used in the context of the Euler or Navier-Stokes equations correspond to ill-posed models. The square of the sound speed becomes negative in the spinodal zone. (ur model considers phase change as a kinetic transformation and not as a thermodynamic one. The phase diagram in figure 7 illustrates both options. With the kinetic 

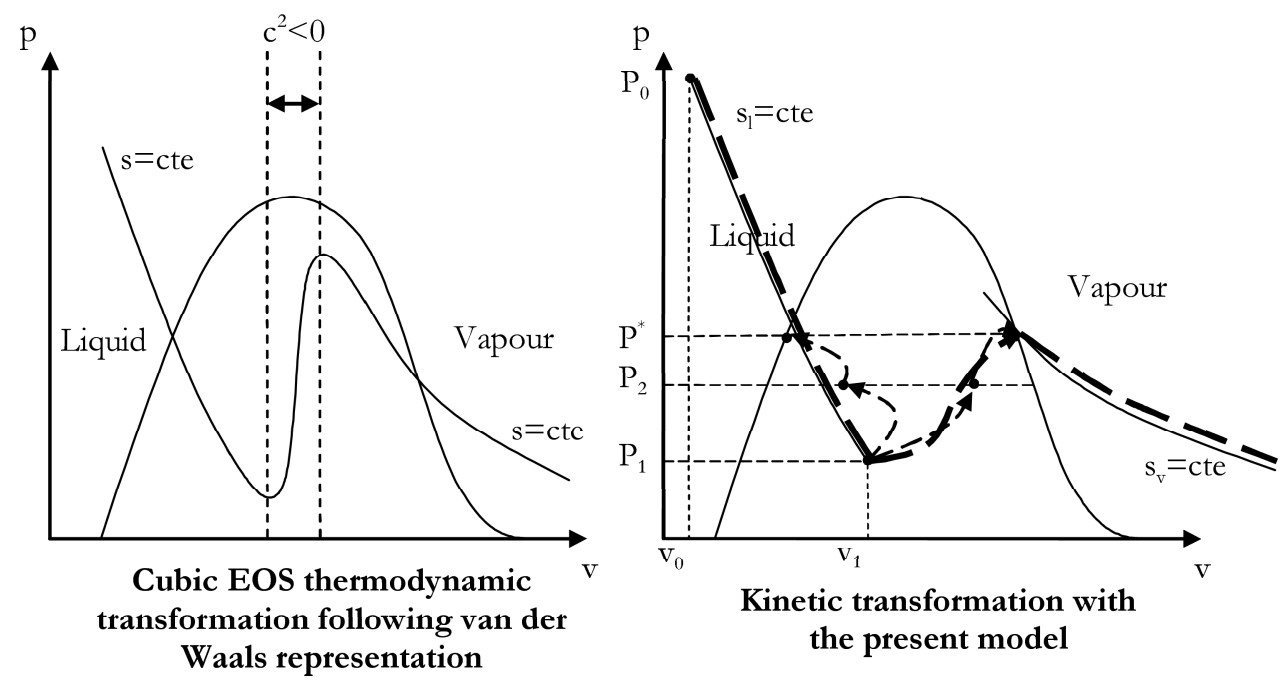

FIGURE 7. Schematic representation of the thermodynamic path using a cubic EOS compared to the kinetic process represented in dashed line. With the cubic EOS, hyperbolicity is lost in the spinodal zone. The present model consists in using a kinetic transformation to connect the isentropes of liquid and vapour. As no thermodynamic path is involved, the mixture sound speed is always defined. In the kinetic approach. from i metastable liquid (end of liquid isentrope) non equilibrium vapour and liquid are produced at constant specific volume for the mixture. Vapour production makes the pressure increase. During kinetic evolıtion the non equilibrium points of liquid and vapour move in direction of saturation curves. At each non equilibrium state pressure equilibrium is assumed. When thermodynamic equilibrium is reached, liquid and vapour states are located on saturation curves. Then, if the specific volume is increased, the equilibrium concentration evolves and as limit case, the vapour expands along an isentrope starting from the saturation curve. Note that when the various non-equilibrium states are omitted, the global transformation path (in bold dash lines). composed of two thermodynamic paths and a kinetic one gives a transformation very closed to the one of van der Waals. The main difference is that ill posedness issues have been removed.

representation, metastable states are present, and the mixture sound speed is always defined. Such approach poses however extra difficulties:

- Determination of the phases EOS. This issue has been examined previously.

- Determination of mass transfer terms.

The system (3.7) describes a compressible two-phase flow mixture in mechanical equilibrium but out of thermal equilibrium. The goal is now to introduce mass transfer effects. The addition of mass transfer modifies the mass equation of each fluid:

$$
\begin{aligned}
& \frac{\partial \alpha_{1} \rho_{1}}{\partial t}+\operatorname{div}\left(\alpha_{1} \rho_{1} \boldsymbol{u}\right)=\rho \dot{Y}_{1} \\
& \frac{\partial \alpha_{2} \rho_{2}}{\partial t}+\operatorname{div}\left(\alpha_{2} \rho_{2} \boldsymbol{u}\right)=-\rho \dot{Y}_{1}
\end{aligned}
$$

where $\rho \dot{Y}_{1}$ represents the mass flux from fluid 2 to fluid 1. Mass transfer is considered with finite rate. It means that it has no dependence with the small parameter $\epsilon$. Expression for this mass flux has to be determined. 
Mass transfer implies changes in the volume fraction. We assume that the volume fraction equation becomes:

$$
\frac{\partial \alpha_{1}}{\partial t}+\boldsymbol{u} \cdot \nabla \alpha_{1}=K \operatorname{div}(\boldsymbol{u})+\frac{\alpha_{1} \alpha_{2}}{\alpha_{2} \rho_{1} c_{1}^{2}+\alpha_{1} \rho_{2} c_{2}^{2}}\left(\frac{\Gamma_{1}}{\alpha_{1}}+\frac{\Gamma_{2}}{\alpha_{2}}\right) Q_{1}+\frac{\rho \dot{Y}_{1}}{\rho_{I}}
$$

where the interfacial density $\rho_{I}$ has to be determined.

The determination of the expressions for mass transfer $\dot{Y}_{1}$ and interface density $\rho_{I}$ is based upon the analysis of the entropy production. To this end, the first step is to determine the entropy equation for each fluid.

\subsection{Determination of the entropy equations of the phases}

The entropy equations are determined as solutions of an algebraic system built on the basis of:

- energy conservation of the mixture.

- pressure equilibrium between phases.

Let us first examine the constraint given by energy conservation to the entropy equations. By using the energy and momentum equations of system (3.7), a simpler form of the energy equation is obtained:

$$
\frac{d e}{d t}+p \frac{d v}{d t}=0
$$

Where the mixture internal energy is defined by $e=Y_{1} e_{1}+Y_{2} e_{2}$ and the mixture specific volume is given by $v=Y_{1} v_{1}+Y_{2} v_{2}$. Thus (5.2) becomes:

$$
Y_{1}\left(\frac{d e_{1}}{d t}+p \frac{d v_{1}}{d t}\right)+Y_{2}\left(\frac{d e_{2}}{d t}+p \frac{d v_{2}}{d t}\right)+\left(h_{1}-h_{2}\right) \dot{Y}_{1}=0 .
$$

Here $h_{k}=e_{k}+p v_{k}$ is the enthalpy of the phase $\mathrm{k}$. By using the Gibbs identity for each phase $\mathrm{k}$, we have:

$$
\frac{d e_{k}}{d t}+p \frac{d v_{k}}{d t}=T_{k} \frac{d s_{k}}{d t}
$$

The mixture energy conservation now becomes:

$$
Y_{1} T_{1} \frac{d s_{1}}{d t}+Y_{2} T_{2} \frac{d s_{2}}{d t}+\left(h_{1}-h_{2}\right) \dot{Y}_{1}=0
$$

This last equation involves the two functions $\frac{d s_{1}}{d t}$ and $\frac{d s_{2}}{d t}$ that we want to determine.

A second equation is provided by the mechanical equilibrium condition:

$$
p_{1}\left(\rho_{1}, s_{1}\right)=p_{2}\left(\rho_{2}, s_{2}\right),
$$

from which we get,

$$
\left(\frac{\partial p_{1}}{\partial \rho_{1}}\right)_{s_{1}} \frac{d \rho_{1}}{d t}+\left(\frac{\partial p_{1}}{\partial s_{1}}\right)_{\rho_{1}} \frac{d s_{1}}{d t}=\left(\frac{\partial p_{2}}{\partial \rho_{2}}\right)_{s_{2}} \frac{d \rho_{2}}{d t}+\left(\frac{\partial p_{2}}{\partial s_{2}}\right)_{\rho_{2}} \frac{d s_{2}}{d t}
$$

The definition of speed of sound and Gruneisen coefficient for each phase enables us to write:

$$
\text { for } k=1,2 \quad\left(\frac{\partial p_{k}}{\partial \rho_{k}}\right)_{s_{k}}=c_{k}^{2} \quad \text { and } \quad\left(\frac{\partial p_{k}}{\partial s_{k}}\right)_{\rho_{k}}=\rho_{k} \Gamma_{k} T_{k}
$$

The mechanical equilibrium condition (5.4) becomes:

$$
c_{1}^{2} \frac{d \rho_{1}}{d t}+\rho_{1} \Gamma_{1} T_{1} \frac{d s_{1}}{d t}=c_{2}^{2} \frac{d \rho_{2}}{d t}+\rho_{2} \Gamma_{2} T_{2} \frac{d s_{2}}{d t}
$$


The equations (5.3) and (5.5) form a system of two equations with the two unknowns functions $d s_{1} / d t$ and $d s_{2} / d t$, and then we get

$$
\left\{\begin{array}{l}
\frac{\alpha_{1} \rho_{1} \alpha_{2} \rho_{2}}{\rho} T_{1}\left(\frac{\Gamma_{1}}{\alpha_{1}}+\frac{\Gamma_{2}}{\alpha_{2}}\right) \frac{d s_{1}}{d t}=Y_{2}\left(c_{2}^{2} \frac{d \rho_{2}}{d t}-c_{1}^{2} \frac{d \rho_{1}}{d t}\right)-\rho_{2} \Gamma_{2}\left(h_{1}-h_{2}\right) \dot{Y}_{1} \\
\frac{\alpha_{1} \rho_{1} \alpha_{2} \rho_{2}}{\rho} T_{2}\left(\frac{\Gamma_{1}}{\alpha_{1}}+\frac{\Gamma_{2}}{\alpha_{2}}\right) \frac{d s_{2}}{d t}=-Y_{1}\left(c_{2}^{2} \frac{d \rho_{2}}{d t}-c_{1}^{2} \frac{d \rho_{1}}{d t}\right)-\rho_{1} \Gamma_{1}\left(h_{1}-h_{2}\right) \dot{Y_{1}}
\end{array}\right.
$$

The next step is to replace the variation $d \rho_{1} / d t$ and $d \rho_{2} / d t$ by variations of the volume fraction and velocity divergence with the help of the mass equations. The system (5.6) now becomes:

$$
\begin{aligned}
\frac{\alpha_{1} \rho_{1} \alpha_{2} \rho_{2}}{\rho} T_{1}\left(\frac{\Gamma_{1}}{\alpha_{1}}+\frac{\Gamma_{2}}{\alpha_{2}}\right) \frac{d s_{1}}{d t} & =Y_{2}\left[\left(\frac{\rho_{1} c_{1}^{2}}{\alpha_{1}}+\frac{\rho_{2} c_{2}^{2}}{\alpha_{2}}\right) \frac{d \alpha_{1}}{d t}-\left(\rho_{2} c_{2}^{2}-\rho_{1} c_{1}^{2}\right) \operatorname{div}(\boldsymbol{u})\right] \\
& -\rho Y_{2}\left(\frac{c_{1}^{2}}{\alpha_{1}}+\frac{c_{2}^{2}}{\alpha_{2}}\right) \dot{Y}_{1}-\rho_{2} \Gamma_{2}\left(h_{1}-h_{2}\right) \dot{Y}_{1} \\
\frac{\alpha_{1} \rho_{1} \alpha_{2} \rho_{2}}{\rho} T_{2}\left(\frac{\Gamma_{1}}{\alpha_{1}}+\frac{\Gamma_{2}}{\alpha_{2}}\right) \frac{d s_{2}}{d t} & =-Y_{1}\left[\left(\frac{\rho_{1} c_{1}^{2}}{\alpha_{1}}+\frac{\rho_{2} c_{2}^{2}}{\alpha_{2}}\right) \frac{d \alpha_{1}}{d t}-\left(\rho_{2} c_{2}^{2}-\rho_{1} c_{1}^{2}\right) \operatorname{div}(\boldsymbol{u})\right] \\
& +\rho Y_{1}\left(\frac{c_{1}^{2}}{\alpha_{1}}+\frac{c_{2}^{2}}{\alpha_{2}}\right) \dot{Y}_{1}-\rho_{1} \Gamma_{1}\left(h_{1}-h_{2}\right) \dot{Y}_{1}
\end{aligned}
$$

Then by using the volume fraction equation (5.1), the entropy equations become functions only of heat exchange, mass transfer and interfacial density $\rho_{I}$ :

$$
\begin{gathered}
Y_{1} \frac{d s_{1}}{d t}=\frac{H\left(T_{2}-T_{1}\right)}{\rho T_{1}}-\frac{\dot{Y}_{1}\left(h_{1}-h_{2}\right)}{\frac{\Gamma_{1} T_{1}}{\alpha_{1}}\left(\frac{\alpha_{1}}{\Gamma_{1}}+\frac{\alpha_{2}}{\Gamma_{2}}\right)} \\
+\frac{\dot{Y}_{1}}{T_{1}\left(\frac{\Gamma_{1}}{\alpha_{1}}+\frac{\Gamma_{2}}{\alpha_{2}}\right)}\left(\frac{\frac{\rho_{1} c_{1}^{2}}{\alpha_{1}}+\frac{\rho_{2} c_{2}^{2}}{\alpha_{2}}}{\rho_{I}}-\left(\frac{c_{1}^{2}}{\alpha_{1}}+\frac{c_{2}^{2}}{\alpha_{2}}\right)\right) \\
Y_{2} \frac{d s_{2}}{d t}=-\frac{H\left(T_{2}-T_{1}\right)}{\rho T_{2}}-\frac{\dot{Y}_{1}\left(h_{1}-h_{2}\right)}{\frac{\Gamma_{2} T_{2}}{\alpha_{2}}\left(\frac{\alpha_{1}}{\Gamma_{1}}+\frac{\alpha_{2}}{\Gamma_{2}}\right)} \\
+\frac{\dot{Y}_{1}}{T_{2}\left(\frac{\Gamma_{1}}{\alpha_{1}}+\frac{\Gamma_{2}}{\alpha_{2}}\right)}\left(-\frac{\frac{\rho_{1} c_{1}^{2}}{\alpha_{1}}+\frac{\rho_{2} c_{2}^{2}}{\alpha_{2}}}{\rho_{I}}+\left(\frac{c_{1}^{2}}{\alpha_{1}}+\frac{c_{2}^{2}}{\alpha_{2}}\right)\right)
\end{gathered}
$$

\subsection{Examination of the phases entropy production}

Entropy equation for each phase (5.8) is composed of three terms. Each of them expresses a physical phenomenon responsible for entropy production :

- The first one is related to heat exchange,

- The second one is associated to mass transfer.

- The last term is associated to the pressure relaxation process associated to mass transfer. Consider a pressure perturbation appearing during mass transfer, see figure 8 . 

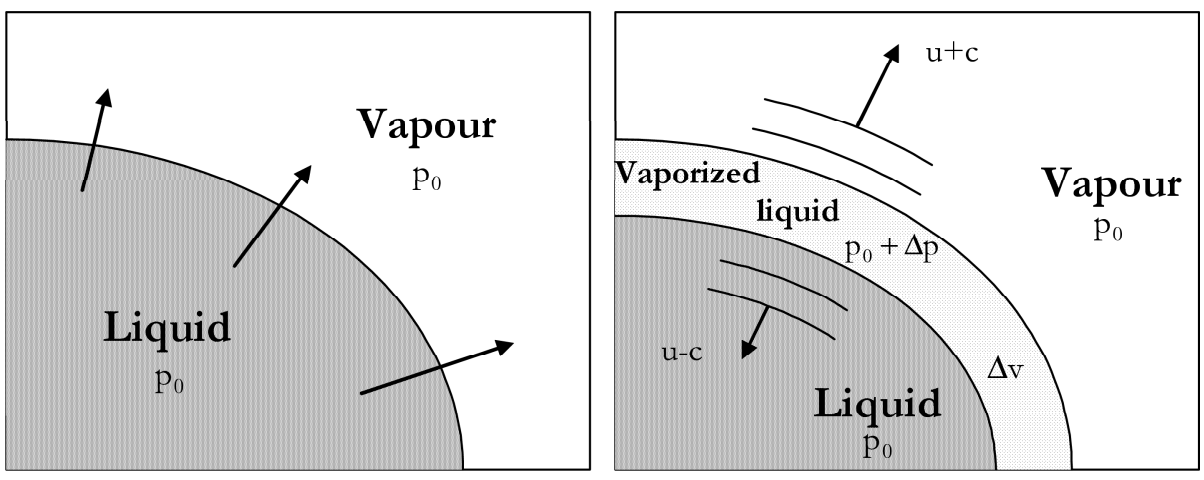

FiguR.F 8. Schematic representation of liquid evaporation. An elementary volume $\Delta v$ of liquid is transformed to vapour with a pressure perturbation. Aconstic waves propagates through liquid and vapour, reflect at volume boundaries and restore pressure equilibrium. The overall process is isentropic as these waves are of small amplitude. These waves are necessarily weak as evaporation is a continuous phenomenon. Elementary volume and pressure perturbations tend to zero.

The system turns back to mechanical equilibrium with the help of acoustic waves emitted during evaporation. This is similar to acoustic waves emitted by flames. These waves of small amplitude are isentropic.

We thus consider that the pressure relaxation process present during mass transfer is isentropic. This corresponds to the third term on the right hand side of (5.8) that vanishes. This remark permits determination of the interface density:

$$
\rho_{I}=\frac{\frac{\rho_{1} c_{1}^{2}}{\alpha_{1}}+\frac{\rho_{2} c_{2}^{2}}{\alpha_{2}}}{\frac{c_{1}^{2}}{\alpha_{1}}+\frac{c_{2}^{2}}{\alpha_{2}}}
$$

\subsection{Mixture entropy inequality}

The second principle of thermodynamics applied to the mixture reads:

$$
\frac{\partial \rho s}{\partial t}+\operatorname{div}(\rho s \boldsymbol{u}) \geqslant 0
$$

where the mixture entropy is defined by $s=Y_{1} s_{1}+Y_{2} s_{2}$.

By using mass equations and expressions (5.8) for the entropies in this inequality leads to:

$$
\frac{H\left(T_{2}-T_{1}\right)^{2}}{\rho}+\left(\bar{g}_{2}-\bar{g}_{1}\right) T_{I} \dot{Y}_{1} \geqslant 0
$$

where an "interface temperature" appears:

$$
T_{I}=\left(\frac{\Gamma_{1} T_{1}}{\alpha_{1}}+\frac{\Gamma_{2} T_{2}}{\alpha_{2}}\right) /\left(\frac{\Gamma_{1}}{\alpha_{1}}+\frac{\Gamma_{2}}{\alpha_{2}}\right)
$$

The extended Gibbs free energies appear:

$$
\text { for } k=1,2 \quad \bar{g}_{k}=h_{k}-\bar{T} s_{k}
$$

with $h_{k}$ the enthalpy of the phase $k$ and $\bar{T}=T_{1} T_{2} / T_{I}$.

It appears clearly that the first term of (5.10) relative to heat exchanges is necessarily 
greater or equal to zero. The second term will be greater or equal to zero if we assume that:

$$
\dot{Y}_{1}=\nu\left(\bar{g}_{2}-\bar{g}_{1}\right)
$$

where $\nu$ is a positive relaxation parameter that controls the rate at which the mixture relaxes to thermodynamic equilibrium. This corresponds to the form of mass transfer terms we were seeking. Note that this modelling of relaxation terms guarantees equilibrium conditions of equal temperatures and equal Gibbs free energy.

\subsection{The model}

We now have a symmetric hyperbolic non-equilibrium compressible two-phase flow model with heat and mass exchanges:

$$
\left\{\begin{array}{l}
\frac{\partial \alpha_{1}}{\partial t}+\boldsymbol{u} \cdot \boldsymbol{\nabla} \alpha_{1}=K \operatorname{div}(\boldsymbol{u})+\frac{\alpha_{1} \alpha_{2}}{\alpha_{2} \rho_{1} c_{1}^{2}+\alpha_{1} \rho_{2} c_{2}^{2}}\left(\frac{\Gamma_{1}}{\alpha_{1}}+\frac{\Gamma_{2}}{\alpha_{2}}\right) Q_{1}+\frac{\frac{\rho_{1} c_{1}^{2}}{\alpha_{1}}+\frac{\rho_{2} c_{2}^{2}}{\alpha_{2}}}{\frac{c_{1}^{2}}{\alpha_{1}}+\frac{c_{2}^{2}}{\alpha_{1}}} \rho \dot{Y}_{1} \\
\frac{\partial \alpha_{1} \rho_{1}}{\partial t}+\operatorname{div}\left(\alpha_{1} \rho_{1} \boldsymbol{u}\right)=\rho \dot{Y}_{1} \\
\frac{\partial \alpha_{2} \rho_{2}}{\partial t}+\operatorname{div}\left(\alpha_{2} \rho_{2} \boldsymbol{u}\right)=-\rho \dot{Y}_{1} \\
\frac{\partial \rho \boldsymbol{u}}{\partial t}+\operatorname{div}(\rho \boldsymbol{u} \otimes \boldsymbol{u})+\nabla p=0 \\
\frac{\partial \rho E}{\partial t}+\operatorname{div}(\boldsymbol{u}(\rho E+p))=0
\end{array}\right.
$$

where:

$$
\begin{aligned}
& K=\frac{\alpha_{1} \alpha_{2}\left(\rho_{2} c_{2}^{2}-\rho_{1} c_{1}^{2}\right)}{\alpha_{2} \rho_{1} c_{1}^{2}+\alpha_{1} \rho_{2} c_{2}^{2}} \\
& \dot{Y}_{1}=\nu\left(\bar{g}_{2}-\bar{g}_{1}\right) \\
& Q_{1}=H\left(T_{2}-T_{1}\right)
\end{aligned}
$$

The mixture pressure is given by (4.4):

$$
p\left(\rho, e, \alpha_{1}, \alpha_{2}, Y_{1}, Y_{2}\right)=\frac{\rho\left(e-Y_{1} q_{1}-Y_{2} q_{2}\right)-\left(\frac{\alpha_{1} \gamma_{1} p_{\infty, 1}}{\gamma_{1}-1}+\frac{\alpha_{2} \gamma_{2} p_{\infty, 2}}{\gamma_{2}-1}\right)}{\frac{\alpha_{1}}{\gamma_{1}-1}+\frac{\alpha_{2}}{\gamma_{2}-1}}
$$

The determination of the temperature relaxation parameter $H$ for a two-phase mixture with arbitrary interfacial area is a difficult issue. The same remark holds for the phase transition kinetics parameter $\nu$ that does not depend only of interfacial area but also of local chemical relaxation. To circumvent these difficulties, we use a solution procedure based on infinite relaxation parameters, but at selected spatial locations only. More precisely, in order to retain metastable states, the relaxation parameters $H$ and $\nu$ will be set to zero for locations far from the interfaces. At the interfaces, they will be taken infinite in order to fulfill equilibrium interface conditions with mass transfer. When dealing 
with interfaces of simple contact, they will be set to zero everywhere. Such procedure is summarized by:

$$
H, \nu=\left\{\begin{array}{cl}
+\infty & \text { if } \epsilon \leqslant \alpha_{1} \leqslant 1-\epsilon \\
0 & \text { otherwise }
\end{array}\right.
$$

\subsection{Limit interface model}

It is interesting to note that this relaxation method corresponds to the local resolution (in fact at the interface only) of the following limit system, corresponding to the mixture Euler equations:

$$
\left\{\begin{array}{l}
\frac{\partial \rho}{\partial t}+\operatorname{div}(\rho \boldsymbol{u})=0 \\
\frac{\partial \rho \boldsymbol{u}}{\partial t}+\operatorname{div}(\rho \boldsymbol{u} \otimes \boldsymbol{u}+p)=0 \\
\frac{\partial \rho E}{\partial t}+\operatorname{div}[(\rho E+p) \boldsymbol{u}]=0
\end{array}\right.
$$

where the mixture density reads $\rho=\alpha_{1} \rho_{1}+\alpha_{2} \rho_{2}$ and the mixture internal energy reads $\rho e=\alpha_{1} \rho_{1} e_{1}+\alpha_{2} \rho_{2} e_{2}$. The total mixture energy is still defined by $E_{2}=e+u^{2} / 2$.

This system is closed by three thermodynamic equilibrium conditions:

$$
\begin{aligned}
& p_{1}=p_{2}=p \\
& T_{1}=T_{2}=T \\
& g_{1}=g_{2} .
\end{aligned}
$$

With the help of the SG EOS (4.1) with conditions (5.13) and (5.14), each phase variable can be expressed as a function of pressure and temperature. The definitions of mixture density and internal energy now read:

$$
\left\{\begin{array}{l}
\rho=\alpha_{2}\left[\rho_{2}(p, T)-\rho_{1}(p, T)\right]+\rho_{1}(p, T) \\
e=\frac{1}{\rho}\left[\alpha_{2}\left[\rho_{2}(p, T) e_{2}(p, T)-\rho_{1}(p, T) e_{1}(p, T)\right]+\rho_{1}(p, T) e_{1}(p, T)\right]
\end{array}\right.
$$

The condition (5.15) now reduces to the mixture EOS (4.3) and the system (5.16) becomes:

$$
\begin{aligned}
\alpha_{2}(T) & =\frac{\rho-\rho_{1}(T)}{\rho_{2}(T)-\rho_{1}(T)} \\
e(T) & =\frac{1}{\rho}\left[\alpha_{2}(T)\left[\rho_{2}(T) e_{2}(T)-\rho_{1}(T) e_{1}(T)\right]+\rho_{1}(T) e_{1}(T)\right] .
\end{aligned}
$$

The equation (5.18) can be solved numerically. It allows the determination of $\alpha_{2}$ with (5.17) and $p$ with (4.3). Thus, the system is closed.

The system (5.12) can also be written under primitive variable form:

$$
\left\{\begin{array}{c}
\frac{d \rho}{d t}+\rho \frac{\partial u}{\partial x}=0 \\
\frac{d u}{d t}+\frac{1}{\rho} \frac{\partial p}{\partial x}=0 \\
\frac{d p}{d t}+\rho c^{2} \frac{\partial u}{\partial x}=0
\end{array}\right.
$$


By using the sound speed definition $c^{2}=(\partial p / \partial \rho)_{s}$ the following expression is obtained:

$$
\frac{1}{\rho c_{e q}^{2}}=\frac{\alpha_{1}}{\rho_{1} c_{1}^{2}}+\frac{\alpha_{2}}{\rho_{2} c_{2}^{2}}+T\left[\frac{\alpha_{1} \rho_{1}}{C_{p, v}}\left(\frac{d s_{1}}{d p}\right)^{2}+\frac{\alpha_{2} \rho_{2}}{C_{p, l}}\left(\frac{d s_{2}}{d p}\right)^{2}\right]
$$

corresponding to the thermodynamic equilibrium mixture speed of sound. Details are given in appendix A. The Wood formula (4.5) is recovered with the first two terms of (5.20). This limit model is again hyperbolic with the characteristic waves speeds: $u+c_{e q}$, $u-c_{e q}$ and $u$.

However, with the algorithm detailed in the following, there is no need to solve explicitly (5.12). The coupling with chemically inert zones far from evaporation fronts and diffuse interface zone governed by (5.12) will be done by the numerical procedure described in the next section. It relies on system (5.11).

\section{Numerical method}

The numerical method to solve the compressible two-phase flow system (5.11) with heat and mass transfer proceeds in two steps. At each time step, the hyperbolic system in absence of heat and mass transfer is solved. This provides the non equilibrium hydrodynamic field. Stiff thermal and chemical relaxations are then solved at the interfaces only. The interfaces are detected from the knowledge of volume fraction fields.

The basic ingredients of the hyperbolic solver are summarized hereafter following Petitpas et al. (2007). This solver is not conventional as the hyperbolic system is not conservative. Conventional Godunov type schemes or other existing methods are not suitable. for its resolution. Then we present a new stiff differential solver specifically derived for the present model. It is used for integration of stiff heat and mass transfer terms.

\subsection{Hyperbolic solver}

The hyperbolic system (5.11) without heat and mass transfer reads:

$$
\left\{\begin{array}{l}
\frac{\partial \alpha_{1}}{\partial t}+\boldsymbol{u} \cdot \nabla \alpha_{1}=K\left(\alpha_{1}, \rho_{1}, \rho_{2}, p\right) \operatorname{div}(\boldsymbol{u}) \\
\frac{\partial \alpha_{1} \rho_{1}}{\partial t}+\operatorname{div}\left(\alpha_{1} \rho_{1} \boldsymbol{u}\right)=0 \\
\frac{\partial \alpha_{2} \rho_{2}}{\partial t}+\operatorname{div}\left(\alpha_{2} \rho_{2} \boldsymbol{u}\right)=0 \\
\frac{\partial \rho \boldsymbol{u}}{\partial t}+\operatorname{div}(\rho \boldsymbol{u} \otimes \boldsymbol{u})+\nabla p=0 \\
\frac{\partial \rho E}{\partial t}+\operatorname{div}[\boldsymbol{u}(\rho E+p)]=0
\end{array}\right.
$$

The volume fraction equation of system (6.1) is not written in conservative form. This rises two important difficulties regarding numerical resolution:

- Conventional shock relations are not available. In absence of these relations, the Riemann problem cannot be solved. The Riemann problem is the cornerstone of modern numerical methods to solve hyperbolic systems.

- The average of the volume fraction variable within a computational cell has no physical sense. Cell averages have sense only for conservative variables. 
Non conventional shock relations for system (6.1) are proposed in Saurel et al. (2007b). These relations:

- Guarantee conservation of the mixture.

- Tend to the single-phase shock relations when one of the phases disappears. They thus guarantee a correct behavior in the single-phase limit. This feature is important for interface problems.

- Preserve volume fraction positivity. This is also an important feature for numerical resolution in the presence of material interfaces.

- Are symmetric with respect to the phases. This allows a possible extension of the model to an arbitrary number of components.

- Are in perfect agreement with experimental shock measurements. It has been validated for more than 100 experimental tests involving a wide range of shock strength, very different acoustic impedance ratios between phases, different initial volume fractions of the phases, different EOS of pure components. See again Saurel et, al. (2007b).

- Last, the mixture Hugoniot curve is tangent to the mixture isentrope. This means that multiphase weak shock waves behave like simple compression waves. This feature is also important for the Riemann problem resolution.

These relations are summarized hereafter (6.2):

$$
\begin{aligned}
& \text { for } k=1,2 \quad Y_{k}=Y_{k}^{0} \\
& \rho(u-\sigma)=\rho_{0}\left(u_{0}-\sigma\right)=m \\
& p-p_{0}+m^{2}\left(v-v_{0}\right)=0 \\
& \text { for } k=1,2 \quad e_{k}-e_{k}^{0}+\frac{p+p_{0}}{2}\left(v_{k}-v_{k}^{0}\right)=0 .
\end{aligned}
$$

Thanks to these relations and Riemann invariants, exact or approximate Riemann solvers can be derived (Petitpas et al. 2007). (Once the Riemann problem is solved, the second difficulty is to average or project the Riemann problem solution onto the computational cell. Because of the volume fraction variable, we cannot use conventional projection methods. Thus a specific relaxation-projection method has been derived in Saurel et al. (2007a) and Petitpas et al. (2007). All details are available in these references.

\subsection{Stiff thermo-chemical solver}

The cell now contains a multiphase mixture in mechanical equilibrium but out of thermal and chemical equilibrium. Indeed, each phase in the cell has its own temperature and Gibbs free energy. In order to fulfill interface conditions in the presence of heat and mass transfer (equal temperatures and chemical potentials) a relaxation method is used. The interface is located by the following procedure:

- A cell is considered filled by a pure fluid when its volume and mass fractions are closed to 1 (say for example $1-\epsilon_{1}$ where typically $\epsilon_{1}=10^{-8}$ ). The interface corresponds to mixture cells when volumes and mass fractions range between $\epsilon_{2}$ and $1-\epsilon_{2}$ (typically, $\epsilon_{2}=10^{-6}$ ). This second small parameter has to be chosen in order that evaporation occur only in the interfacial zone and not during expansion waves that produce metastable states. Expansion waves indeed induce gas volume fraction increase. If $\epsilon_{2}$ is taken too close of $\epsilon_{1}$ evaporation may occur too early and not only in the interfacial zone.

- Mass transfer is allowed if one of the fluid in the mixture cell is metastable $\left(T_{k}>\right.$ $\left.\left.T_{\text {sat }}(P)\right)\right)$ 
The hydrodynamic evolution being now achieved by the numerical approximation of system (6.1) the goal is now to solve the following system of ordinary differential equations at the interface only:

$$
\left\{\begin{array}{l}
\frac{\partial \alpha_{1}}{\partial t}=\frac{\alpha_{1} \alpha_{2}}{\alpha_{2} \rho_{1} c_{1}^{2}+\alpha_{1} \rho_{2} c_{2}^{2}}\left(\frac{\Gamma_{1}}{\alpha_{1}}+\frac{\Gamma_{2}}{\alpha_{2}}\right) Q_{1}+\frac{\frac{\rho_{1} c_{1}^{2}}{\alpha_{1}}+\frac{\rho_{2} c_{2}^{2}}{\alpha_{2}}}{\frac{c_{1}^{2}}{\alpha_{1}}+\frac{c_{2}^{2}}{\alpha_{1}}} \rho \dot{Y}_{1}=S_{\alpha_{1}} \\
\frac{\partial \alpha_{1} \rho_{1}}{\partial t}=\rho \dot{Y}_{1}=S_{Y_{1}} \\
\frac{\partial \alpha_{2} \rho_{2}}{\partial t}=-\rho \dot{Y}_{1} \\
\frac{\partial \rho \boldsymbol{u}}{\partial t}=0 \\
\frac{\partial \rho E}{\partial t}=0
\end{array}\right.
$$

The integration of this system necessitates closure relations for $Q_{1}$ and $\dot{Y}_{1}$, in particular regarding relaxation parameters $h$ and $\nu$. In order that the model be free of parameters we assume that thermodynamic local equilibrium is reached at the interface at any time. It means that $h$ and $\nu$ tend to infinity locally. This assumption is standard at equilibrium interfaces when mass transfer occurs. It does not mean that the entire flow evolves at thermodynamic equilibrium. The states remain out of equilibrium far from interfaces.

In the context of numerical integration, taking infinite relaxation parameters means that the equilibrium has to be reached at the end of each time step. The time step is imposed by CFL restriction of the hydrodynamic system. Using this time step, we. determine $Q_{1}$ and $\dot{Y}_{1}$ in order that thermodynamic equilibrium be reached at the end of each time step. To determine these source terms, the equations for the temperatures and Gibbs free energies differences are necessary. They read:

$$
\left\{\begin{aligned}
\frac{\partial \Delta T}{\partial t} & =A Q_{1}+B \dot{Y}_{1} \\
\frac{\partial \Delta g}{\partial t} & =A^{\prime} Q_{1}+B^{\prime} \dot{Y}_{1}
\end{aligned}\right.
$$

where $A, B, A^{\prime}, B^{\prime}$ are functions of all flow variables. Their expressions are detailed in appendix $\mathrm{B}$.

The simplest numerical approximation of these equations is used. Let " $n$ " and " $n+1$ " denote two successive time steps. The variables at time $t^{n}$ are taken equal to those resulting of the numerical integration of system (6.1). The variables at time $t^{n+1}$ denote the end of the integration process, including both hydrodynamic effects and source terms (6.3). The simplest numerical approximation of this ()DE system writes:

$$
\left\{\begin{array}{l}
\frac{(\Delta T)^{n+1}-(\Delta T)^{n}}{\Delta t}=A^{n} Q_{1}^{n}+B^{n} \dot{Y}_{1}^{n} \\
\frac{(\Delta g)^{n+1}-(\Delta g)^{n}}{\Delta t}=A^{\prime n} Q_{1}^{n}+B^{\prime n} \dot{Y}_{1}^{n}
\end{array}\right.
$$


By imposing that thermodynamic equilibrium is reached at the end of the time step we have: $(\Delta T)^{n+1}=0$ and $(\Delta g)^{n+1}=0$. The corresponding heat and mass transfer terms are given by:

$$
\left\{\begin{array}{l}
Q_{1}=-\frac{B^{\prime}}{A B^{\prime}-A^{\prime} B} \frac{(\Delta T)^{n}}{\Delta t}+\frac{B}{A B^{\prime}-A^{\prime} B} \frac{(\Delta g)^{n}}{\Delta t} \\
\dot{Y}_{1}=\frac{A^{\prime}}{A B^{\prime}-A^{\prime} B} \frac{(\Delta T)^{n}}{\Delta t}-\frac{A}{A B^{\prime}-A^{\prime} B} \frac{(\Delta g)^{n}}{\Delta t}
\end{array}\right.
$$

These approximations of heat and mass transfer terms allow the calculation of source terms $S_{\alpha_{1}}$ and $S_{Y_{1}}$ of system (6.3). Nevertheless, there is no guarantee that positivity of the solution be preserved, in particular regarding mass and volume fractions. A limitation of these source terms has to be done. Let us express the maximum admissible source term for the volume fraction evolution in order to preserve positivity of this variable:

$$
S_{\max , \alpha_{1}}= \begin{cases}\frac{1-\alpha_{1}}{\Delta t} & \text { if } \quad S_{\alpha_{1}}>0 \\ \frac{-\alpha_{1}}{\Delta t} & \text { otherwise. }\end{cases}
$$

The same maximum source term is computed for mass fraction positivity. Thus, if $\left|S_{\max , \alpha_{1}}\right|>\left|S_{\alpha_{1}}\right|$ and $\left|S_{\max , Y_{1}}\right|>\left|S_{Y_{1}}\right|$; the numerical integration can be done with the hydrodynamics time step. Otherwise, equations are stiff and the integration time. step has to be reduced. The ratio $R_{\alpha_{1}}=S_{\max , \alpha_{1}} / S_{\alpha_{1}}$ is computed and the system (6.3) is integrated on a fraction of the time step, typically: $\Delta t_{\text {chem }}=R_{\alpha_{1}} \Delta t / 2$. Successive point integrations are done in order that the complete hydrodynamic step be covered.

With this algorithm, thermodynamic equilibrium is reached very fast and positivity of the solution is preserved. Using this relaxation method corresponds to the local resolution (actually at the interface only) of the limit system, corresponding to the mixture Euler equations (5.12).

\section{Numerical Results.}

The aim of this section is to highlight model's capabilities for the numerical resolution of interface problems with or without mass transfer. A set of one dimensional tests are first considered for validation issues. Then two-dimensional tests are secondly addressed for the dynamic creation of super-cavitating flow pockets.

\subsection{Two-phase shock tube without mass transfer.}

In this example, the left part of a shock tube is filled with liquid dodecane at high pressure $p_{l}=10^{8} \mathrm{~Pa}$ with density $\rho_{l}=500 \mathrm{~kg} / \mathrm{m}^{3}$. The right chamber is set at atmospheric pressure and filled with vapor dodecane at density $\rho_{v}=2 \mathrm{~kg} / \mathrm{m}^{3}$. The initial discontinuity is located at $x=0.75 \mathrm{~m}$ in a $1-\mathrm{m}$ length tube. For numerical reasons, each chamber of the tube contains a weak volume fraction of the other fluid (typically $10^{-8}$ ).

In the first example, the liquid - gas interface is solved as a contact discontinuity: heat and mass transfer are removed. The results are shown at time $t=473 \mu \mathrm{s}$ in figure 9 and consist in three conventional waves. From left to right, a left-facing rarefaction wave propagates through the liquid, the contact discontinuity is moving from left to right and a right-facing shock propagates through dodecane vapor. The numerical solution is compared to the exact one and shows a perfect agreement. 

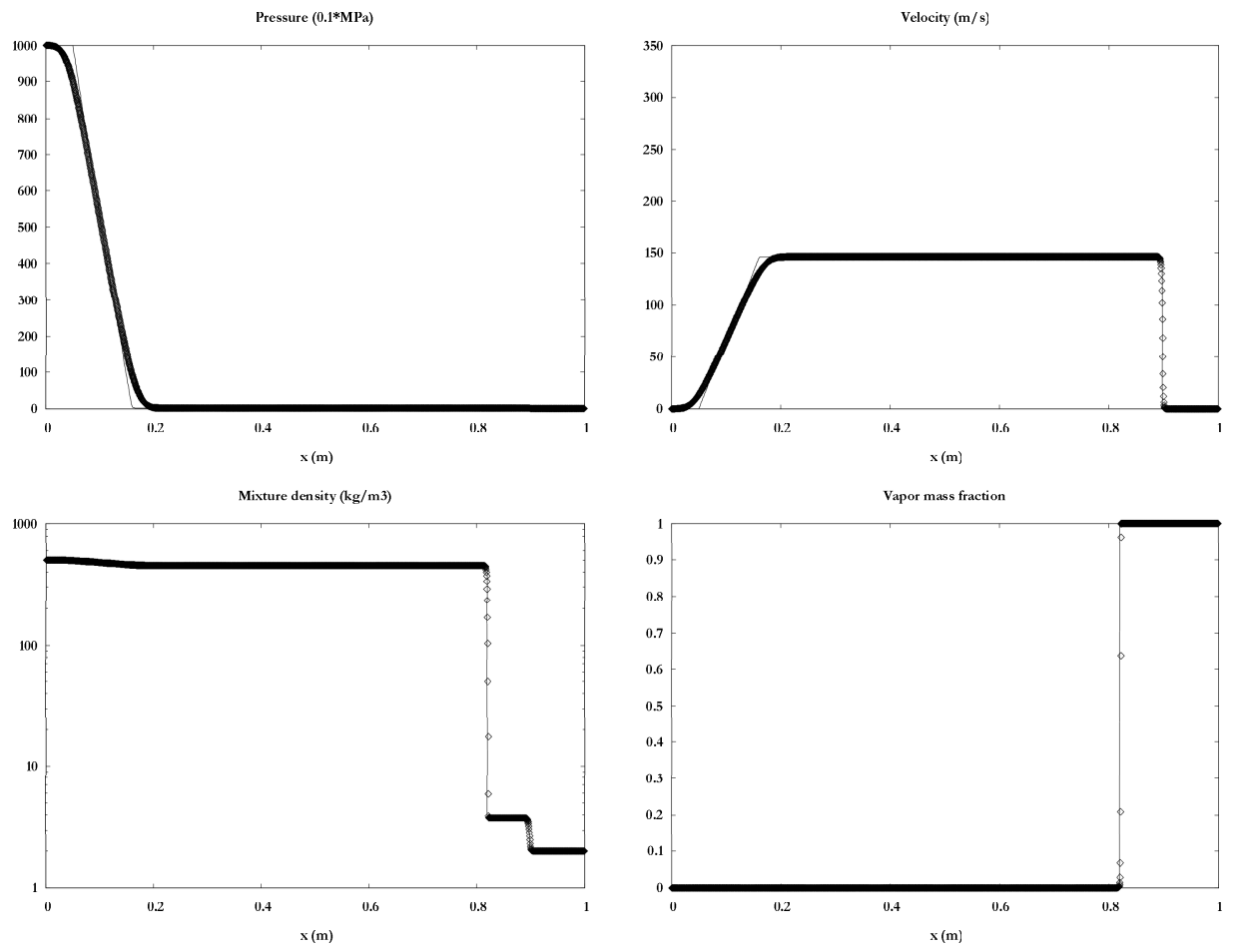

Figur. 9. Dodecane liquid-vapor shock tube without mass transfer. The numerical solution (symbols) is compared to the exact one (lines). An excellent agreement is observed. The velocity graph scale has been chosen in order that a direct comparison with the results of the next figure be easy.

\subsection{Two-phase shock tube with mass transfer.}

We now rerun the same test case and consider heat and mass transfer at the interface. Indeed, the rarefaction wave propagation transforms the stable high pressure liquid dodecane into is superheated liquid and evaporation has to be considered (figure 10). An additional left-facing wave (evaporation front) appears between the rarefaction wave and the contact discontinuity. It propagates through the superheated liquid and produces a liquid-vapor mixtıre at thermodynamic equilibrium and high velocity.

Infinite relaxation parameters are used at the interface only. The limit model corresponds to the mixture Euler equations (5.12), for which acoustic disturbances propagate at $c_{e q}$ (5.20). Far from the interface, the Kapila et al. (2001) model is solved in absence. of relaxation terms. This model tends to the considered pure fluid Euler equations, for which acoustic disturbances propagate at $c_{k}$ (the speed of sound of phase $k$ ). As $c_{e q}$ is always lower (or equal) than $c_{k}$, acoutic perturbations in pure phases propagates faster than evaporation fronts (whose velocity cannot exceed $c_{e q}$ ). Thus, acoustic precursors are present and correspond to expansion or compression waves in pure fluid. They produce metastable states.

We c:an see the four waves (the left facing expansion wave, the evaporation front, the contact discontinuity and the right-facing shock as was mentioned in the introduction section and in figure 3 ) on the mixture density graph of figure 10 . The evaporation front makes the vapor mass fraction increase, but total evaporation is not reached. The second jump in mass fraction is related to the contact discontinuity. 

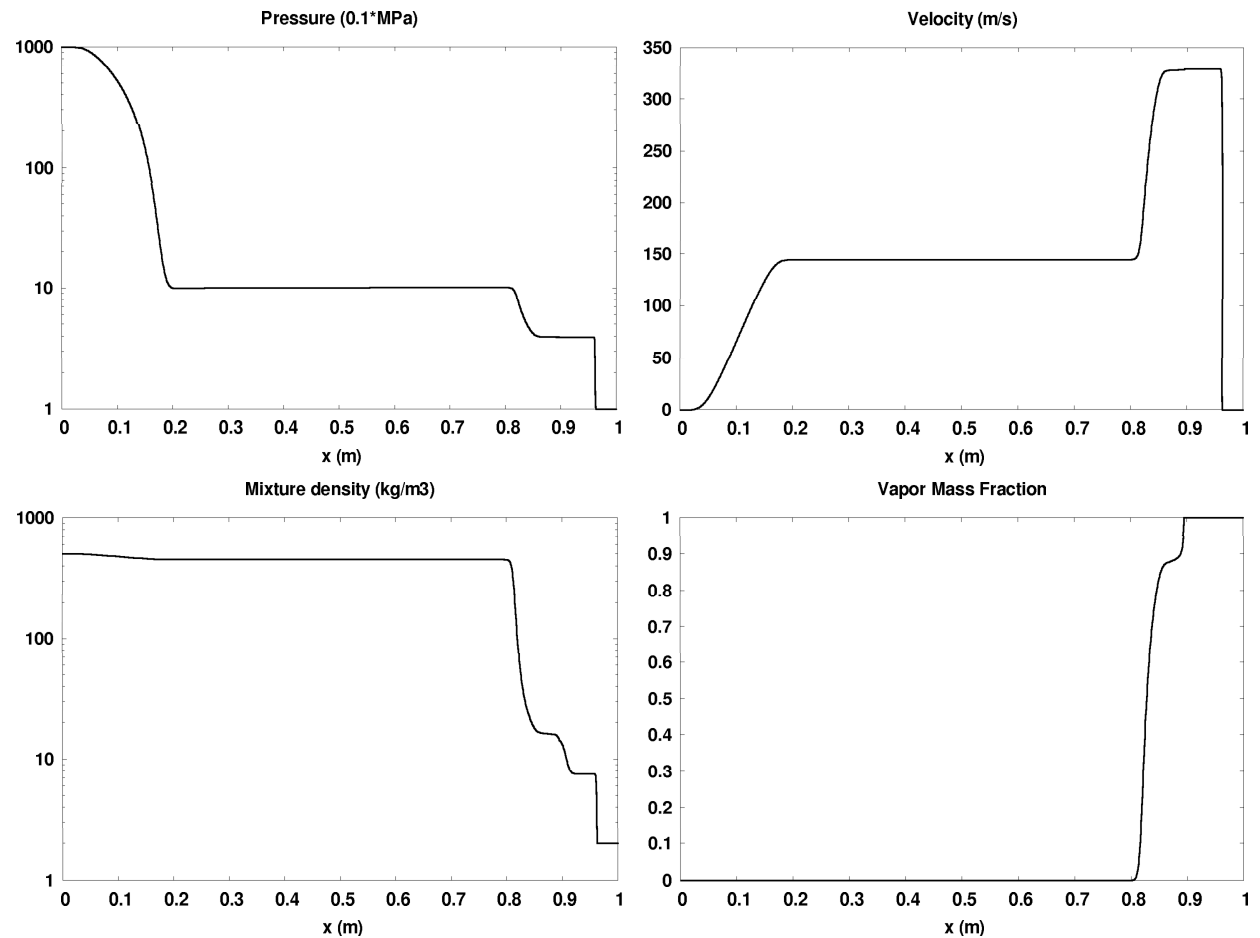

FiguR.F 10. Dodecane liquid-vapor shock tube with mass transfer. The thermo-chemical solver is used at the interface. An extra wave appears traducing evaporation of superheated liquid. The second jump in mass fraction is the contact discontinuity between liquid-vapor mixture produced by evaporation and shocked vapor initially present in the right chamber. The velocity graph can be compared with those of the preceding figure, where mass transfer at the interface. is absent.

The corresponding phase-space trajectory is reported in figure 11 together with the mixture density graph where all fluid states are visible. Stable high pressure liquid dodecane. represented by point 1 is expanded until point 2 with an isentropic path. Point 2 corresponds to metastable liquid dodecane. The evaporation front transforms the metastable. liquid into a liquid-vapor mixture under thermodynamic equilibrium (points $3_{l}$ and $3_{v}$ ). There is no thermodynamic connection between point 2 and points $3_{l}$ and $3_{v}$ because they are just linked by a kinetic process. (On the other part of the shock tube, initial vapor (point 5) is shocked and follows a Hugoniot curve until point 4 . Points 3 and 4 have no thermodynamic connections as they are just linked by mechanical equilibrium through a simple contact discontinuity.

\subsection{Validation against shock tube experiments.}

Experiments in shock tube have been carried out by Simoes-Moreira \& Shepherd (1999). Liquid dodecane is initially kept stable for a certain temperature and is suddenly expanded into a low pressure chamber. An evaporation front propagates into metastable. liquid dodecane ejecting high velocity liquid-vapor mixture. Velocity of this front was measured for different initial temperatures of liquid dodecane. Front velocities are computed under the same conditions with the present model and compared with experiments. Results are shown in figure 12. Each point corresponds to a shock tube computation with a given initial temperature of the liquid in the high pressure chamber. The agreement is not perfect but front velocities are of the same order of magnitude and the same ten- 

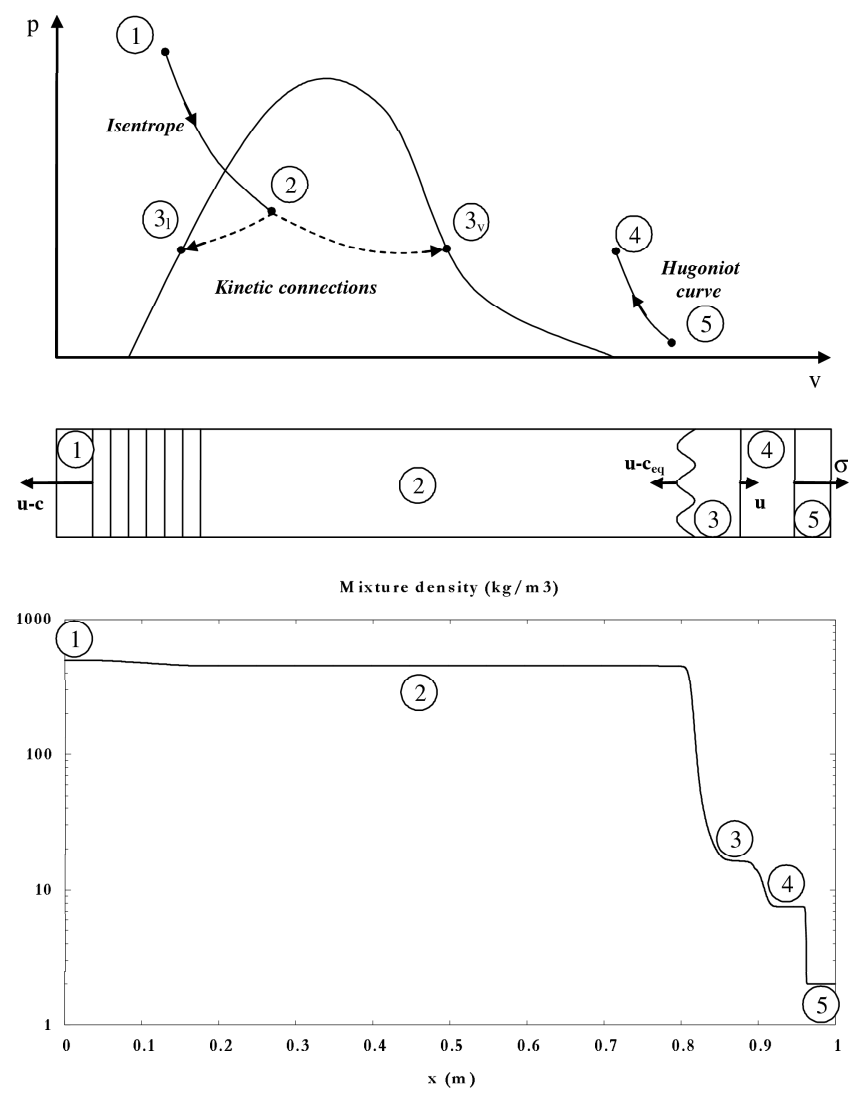

Figure 11. Phase-space trajectory for the flow simulated in figure 10. Trajectories in dashed lines (between points 2 and 3 ) in the phase diagram represent kinetic connections between superheated liquid and equilibrium mixtıre. Points 3 and 4 have no thermodynamic connections as they are just linked by mechanical equilibrium through a simple contact discontinuity separating vaporized mixture and shocked initial vapor.

dency of increasing front velocity with increasing temperature is observed. The slight discrepancies between experimental and computed results are explained by the inaccuracies present in the simplified model EOS used for the phases. Using a more sophisticated EOS than SG (Mie Gruneisen for example) is an option to improve this point.

\subsection{About the C.J kinetic relation.}

The C.J kinetic relation is often used to close Rankine Hugoniot system to deal with evaporation fronts in metastable liquids. In this context, the front is considered as a discontinuity whose speed is determined by the C.J condition. Obviously, such procedure is approximate and valid only in limit situations, when the liquid is highly metastable so that the system tends to evaporate as fast as possible i.e. with the maximum admissible mass flow rate. This limit evaporation regime is considered in the present model with slight modification.

In the present model the interface is solved as a relaxation zone where the multiphase flow model tends to the equilibrium Euler equations. This last system admits very different waves speeds from the ones of the temperature non equilibrium model. This results in the appearance of a transition front that corresponds to an expansion wave of the equilibrium model. Indeed, observation of the numerical results at different times show 
Front velocity $(\mathrm{m} / \mathrm{s})$

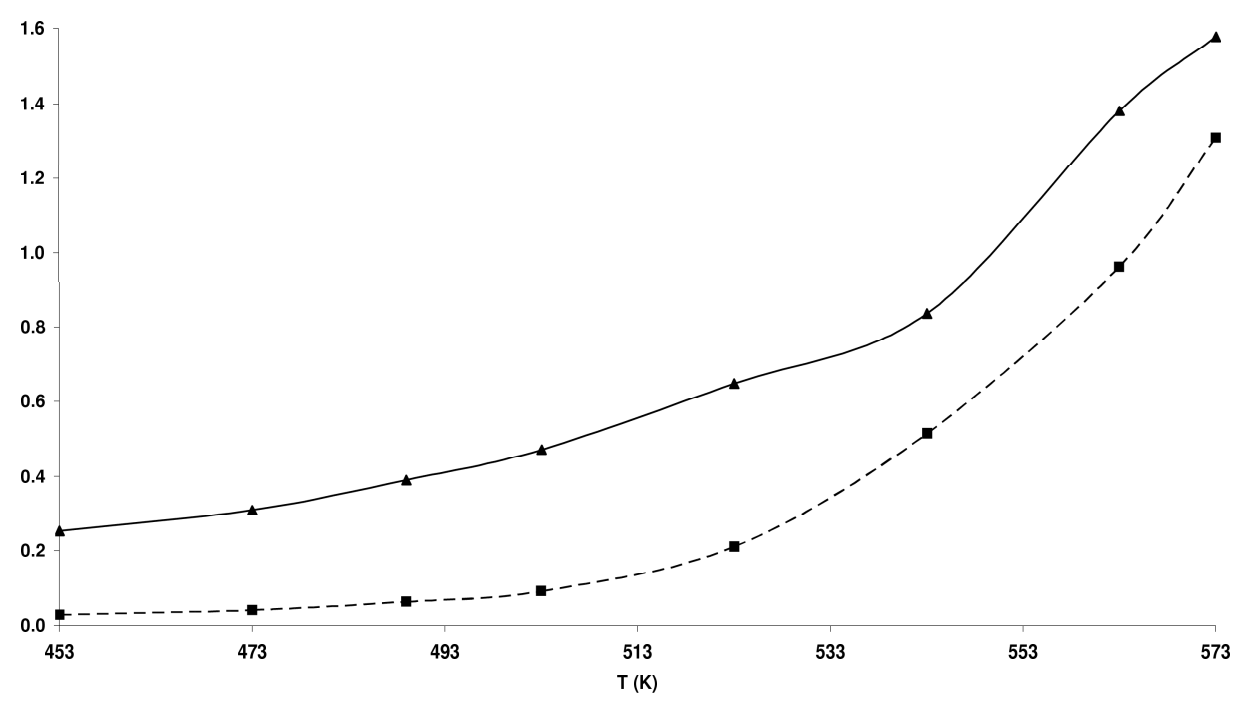

Figus. 12. Evaporation front velocity in superheated dodecane versus initial temperature of liquid dodecane. The experimental results of Simoes-Moreira \& Shepherd (1999) (line) are compared with front velocities computed with the new model (dash line).

a smearing of the evaporation wave. exactly as a computed expansion wave and not as a contact discontinuity. As represented in the figure 13, this expansion wave propagates to the left at the velocity approximately equal to $u-c_{e q}$. There is no contradiction between our modelling and preceding Hugoniot analysis. Conventional modelling based on jump conditions closed by CJ assumption consider the front as a discontinuity obeying the principle of mass, momentum and energy conservation with an evaporation wave propagating at the maximum admissible speed (CJ). Such model was proposed by Chaves (1984) and validated against many experiments when a retrograde liquid is highly metastable. In such modelling, negative pressure jumps are obtained ('negative shocks' (Thompson \&. Lambrakis 1973)). In our approach, the wave is solved as an expansion wave of the relaxed system. It also obeys to the principles of mass, momentum and energy conservation. Its speed corresponds to the one of acoustic waves of the equilibrium system $u-c_{e q}$ with the sound speed given by (5.20). It is also associated to a pressure decrease. as in all expansions waves. It is thus in perfect agreement with preceding analysis, except that the front is not considered as a discontinuity.

These observations give an interpretation of the model proposed by Chaves (1984) where the Chapmann Jouguet kinetic relation ( $\sigma=u \pm c$ where $\sigma$ represents the front velocity) was proposed. The present results show that this kinetic relation is appropriate in such limit situation and that the evaporation front corresponds to an expansion wave of the thermodynamic equilibrium model.

The differences in accuracy when compared for example with Simoes-Moreira \& Shepherd (1999) results are only due to the EOSs. The equilibrium EOS of the present relaxed system is not exactly the same as in Simoes-Moreira \& Shepherd (1999). The E()S used by these authors is more accurate, but not valid everywhere in particular in the spinodal zone.

Let us mention that CJ evaporation fronts are associated to extreme conditions. Under 


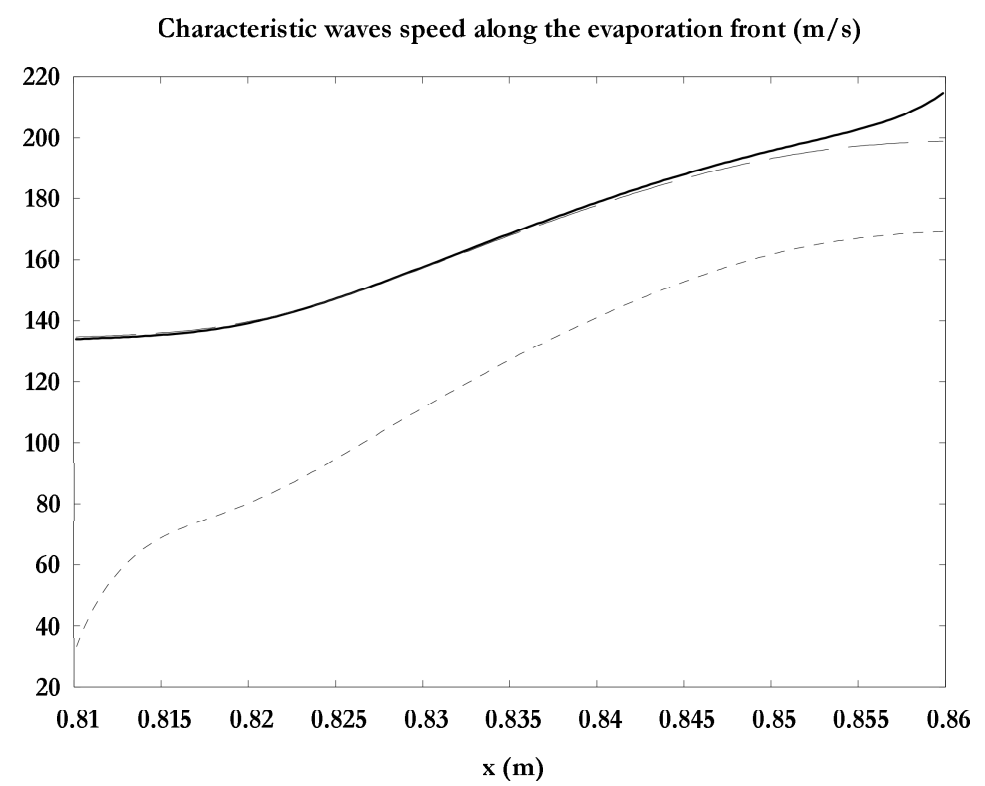

FIgURE 13. Comparison between local wave speed (lines) computed with $\sigma_{i}=\frac{(\rho u)_{i}-(\rho u)_{i-1}}{\rho_{i}-\rho_{i-1}}$, characteristic velocity based on the equilibrium speed of sound $u-c_{e q}$ in dash lines and characteristic velocity based on the mechanical equilibrium $u-c_{W}$ (Wood) speed of sound in dotted lines. The comparison is done for the flow simulated in figure 10 in the x-range where the evaporation front is present. It appears clearly that the evaporation front propagates at CJ speed.

moderate conditions, the front is subsonic and its dynamics is governed by multidimensional effects where thermal diffusion, capillary effects and chemical relaxation play an important role. Such effects can be introduced in the present approach by coupling the present relaxation method with the capillary and dissipative effects modelling described in Perigaud \&. Saurel (2005). Extra efforts are necessary to reach this goal.

\subsection{Two-phase expansion tube.}

In this example, a 1-m length tube is filled with liquid water at atmospheric pressure and with density $\rho=1150 \mathrm{~kg} / \mathrm{m}^{3}$. An initial velocity discontinuity is located at $x=0.5 \mathrm{~m}$. ()n the left, the velocity is set to $u=-2 \mathrm{~m} / \mathrm{s}$ and on the right, $u=2 \mathrm{~m} / \mathrm{s}$. An initial weak volume fraction of vapor water $\left(\alpha_{v}=10^{-2}\right)$ is added to the liquid. Thermodynamic parameters of water are given in table 1. First, the numerical solution without mass transfer is compared to the exact one. The solution is represented in figure 14 at time $t=3.2 \mathrm{~ms}$. It results in left- and right-facing rarefaction waves. The vapor volume. fraction increases at the center of the domain. This is due to the gas mechanical expansion present in small proportions. This effect tends to create new interfaces in the domain. Dynamic appearance of these interfaces is considered in the numerical resolution as well as in the exact solution, provided in Petitpas et al. (2007). The gas pocket that appears is not due to mass transfer, but to mechanical relaxation only (bubbles growth).

However, these rarefaction waves make the liquid metastable and phase transition may occur. Figure 15 presents the solution when mass transfer is involved. The solution is shown at the same time as previously and compared to the previous exact solution without evaporation. Liquid water is expanded until the saturation pressure is reached (see the pressure graph) then evaporation appears and a quite small amount of vapor 

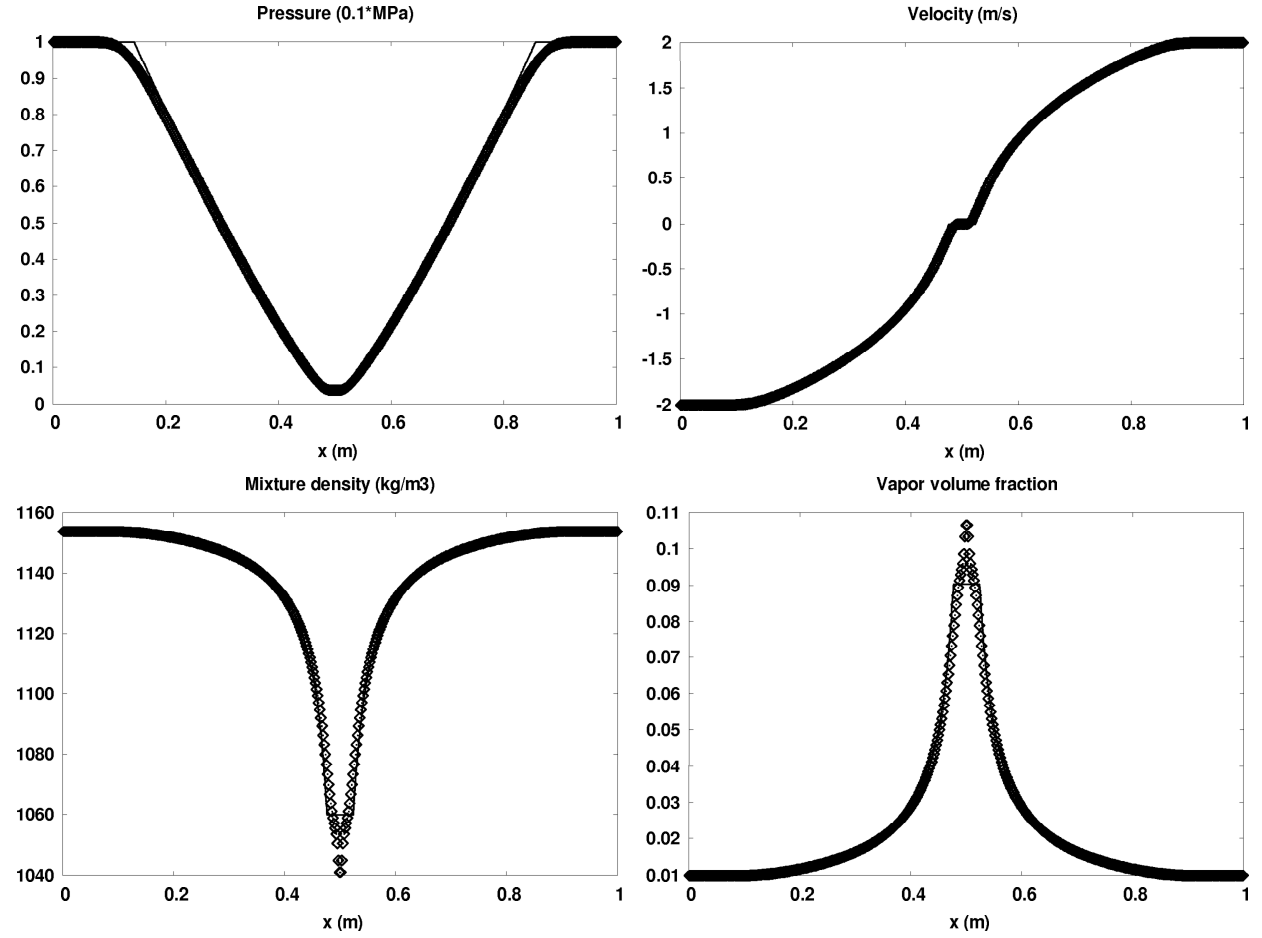

FiguRF 14. The multiphase model computed solution (symbols) withont mass transfer is compared to the exact one (lines) on the symmetric expansion tube.

is created (see the mass fraction graph). This small amount of vapor has nevertheless strong consequences on the vapor volime fraction that increases significantly.

The solution with mass transfer is composed of 4 expansion waves, as shown in figure 16. In order to highlight the two slow expansion waves, the solution is now shown at time $t=5.9 \mathrm{~ms}$ in figure 17 . The two leading fast expansion waves have left the tube. The two slow evaporation fronts have characteristic profiles of expansion waves. This observation confirms the previous interpretation of evaporation fronts as expansion waves of the equilibrium system. When rarefaction effects become stronger. it is possible to see the four waves present on a single graph. In figure 18 the same conditions are used except regarding velocities which are set to $u=-50() \mathrm{m} / \mathrm{s}$ on the left, and $u=5(0) \mathrm{m} / \mathrm{s}$ on the right. In this case, evaporation is much intense resulting in a large cavitation pocket where the gas volume fraction is closed to 1 . However, this pocket does not contain pure gas but a mixture at thermodynamic equilibrium as shown on the mass fraction profile. The various expansion waves have now comparable velocities, as shown in figure 19.

\subsection{Two-dimensional illustrations.}

The model capabilities are now illustrated on severe test problems involving cavitation pockets with or without evaporation. The first example is related to supercavitation around a high velocity underwater projectile. The second test is related to cavitation pockets in fuel injector nozzles.

\subsubsection{High velocity underwater projectile.}

A liquid water flow at velocity $600 \mathrm{~m} / \mathrm{s}$ is considered around an immersed obstacle. Liquid water is initially at atmospheric pressure with a density of $1150 \mathrm{~kg} / \mathrm{m}^{3}$. A weak 

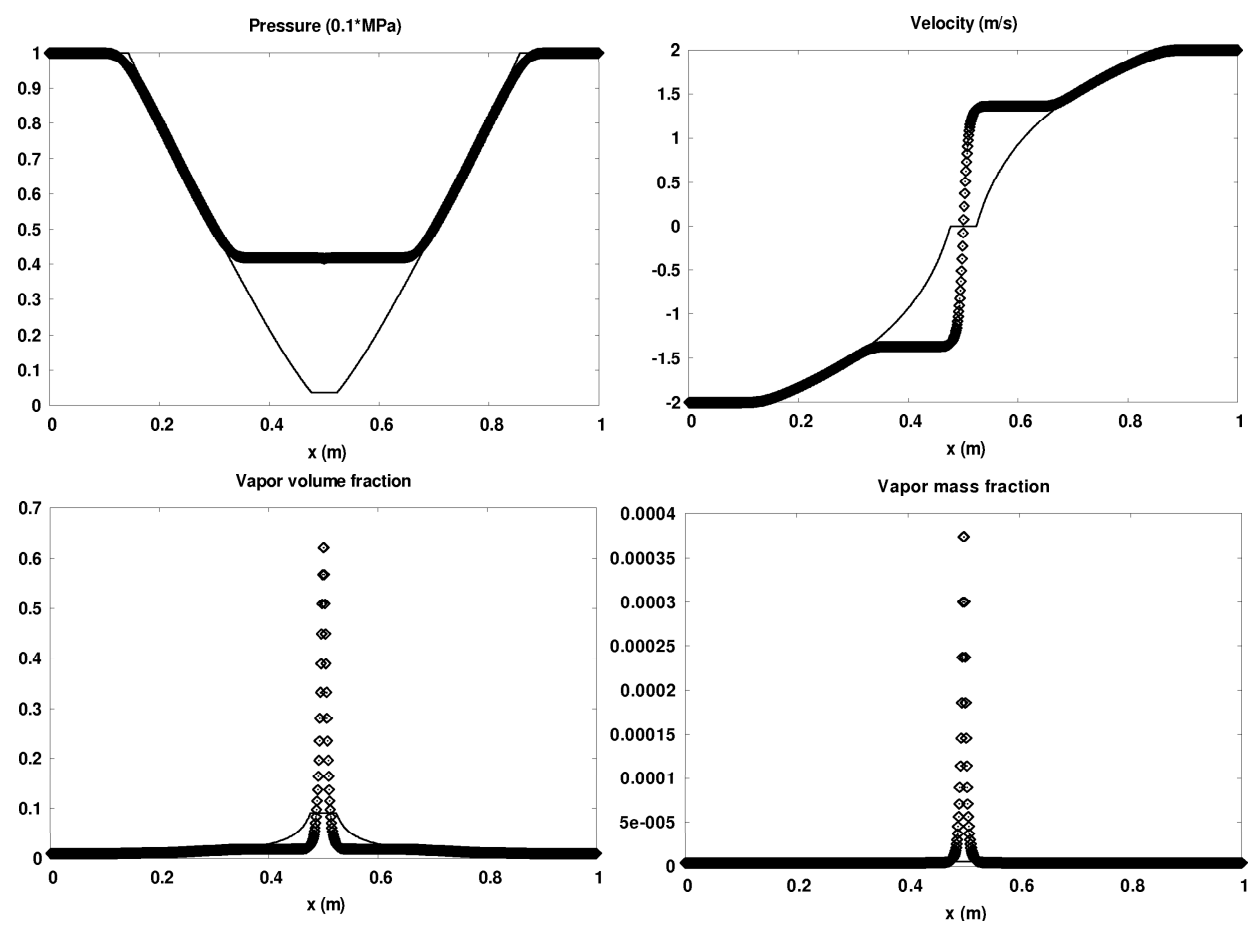

FiguR.F 15. The multiphase model computed solution (symbols) with mass transfer is compared to the exact one (lines) without mass transfer on the symmetric expansion tube. Mass transfer appears and results in important differences for all flow variables.

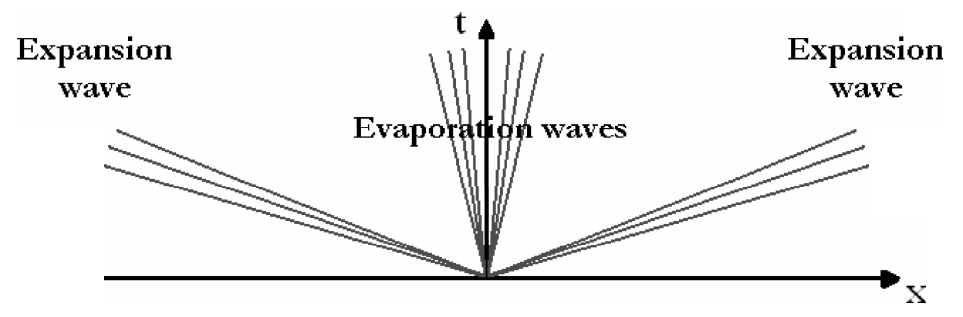

FiguR.F 16. Wave propagation diagram of the symmetric expansion tube.

volume fraction of vapor $\left(\alpha_{v}=10^{-3}\right)$ is initially present in water. At the leading edge of the obstacle a strong compression zone is present and the pressure exceeds $2000 \mathrm{~atm}$. Then the high velocity liquid flow undergoes strong rarefaction waves at each geometrical singularity and the pressure decreases.

()n the left column of figure 21 the computational results are shown at steady state without mass transfer. In absence of mass transfer, the simulation does not breakdown as a small volume fraction of gas is present everywhere. This is a nice feature of Kapila et al. (2001) model. This model however need specific numerical scheme as it is nonconservative. Appropriate numerical method is developed in Petitpas et al. (2007).

On the right of the same figure, mass transfer is involved. Qualitatively, both results are very similar: a cavitation wake appears. But the mechanisms responsible for these cavitation waves are very different. On the left, only mechanical effects are responsible for bubble growth while on the right mechanical and chemical relaxation effects are responsible of the pocket growth. It means that results without mass transfer can be used 

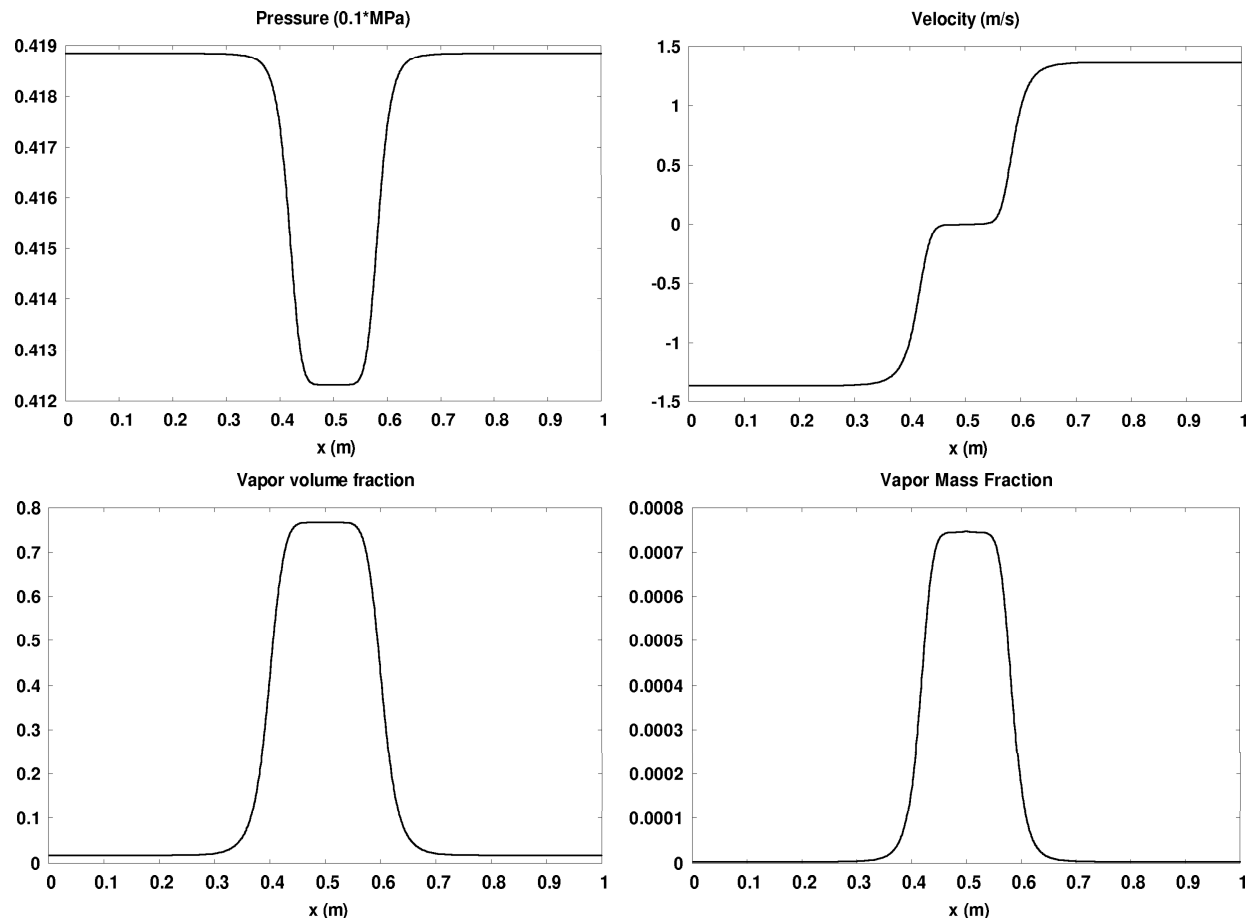

Figur.f 17. Long time behaviour for the test problem of figure 15. The two slow expansion waves are clearly visible.
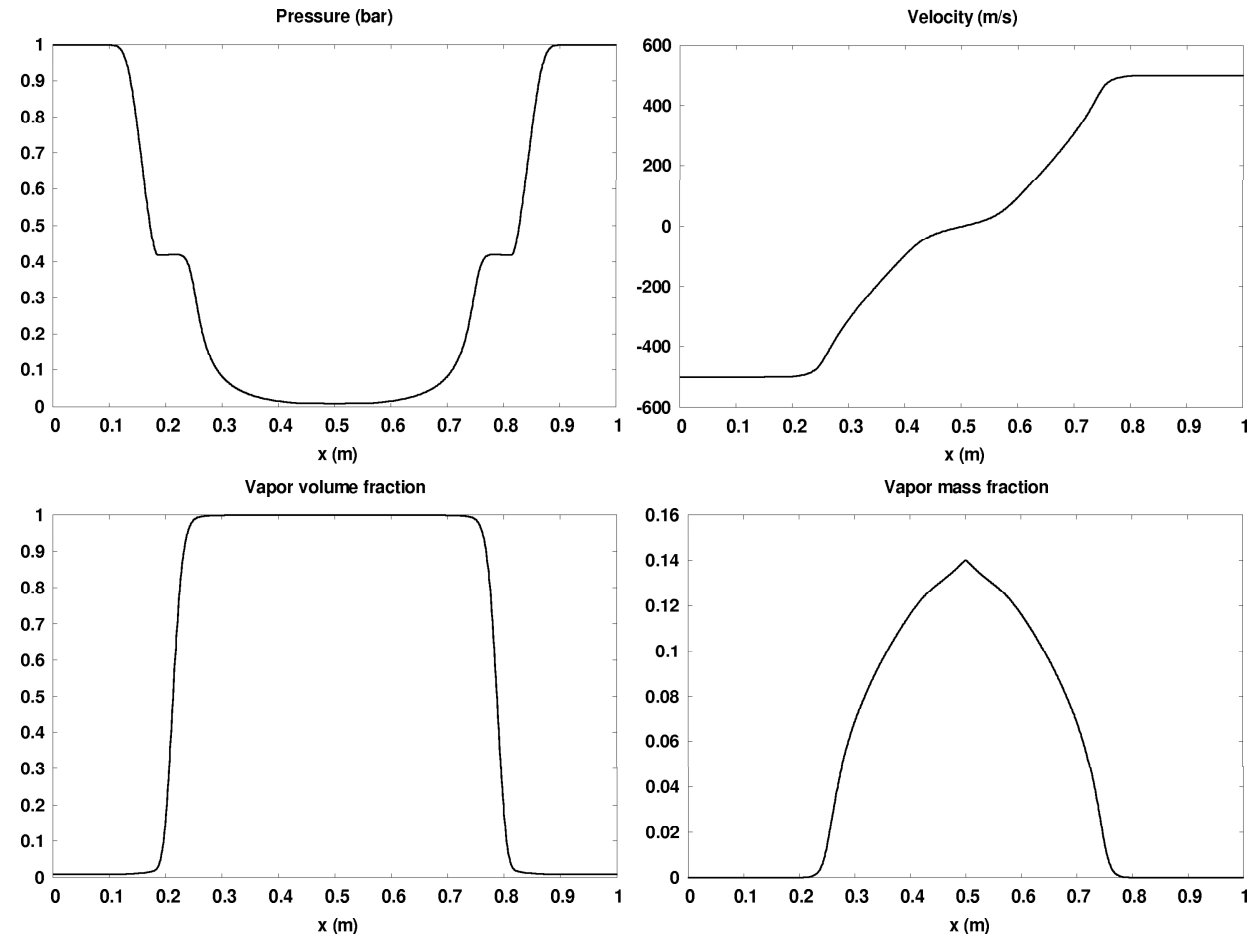

FiguR.F 18. Numerical solution of the multiphase flow model with mass transfer and strong velocity difference in the expansion tube. The four expansion waves are clearly visible. 


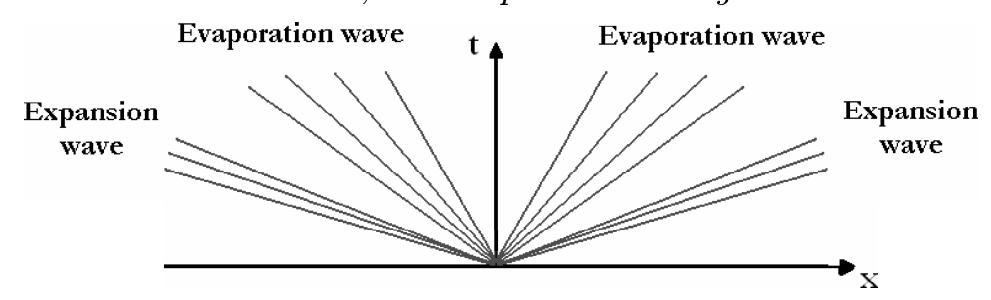

Figure 19. Schematic $(\mathrm{x}, \mathrm{t})$ diagram of the strong symmetric expansion tube.

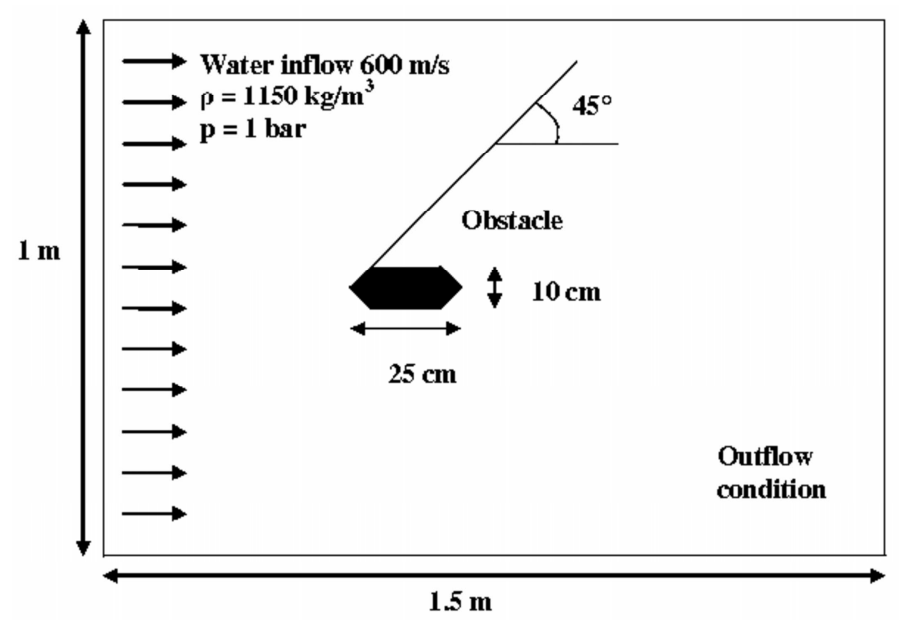

FiguRE 20. Initial configuration of an high velocity underwater projectile.

for a qualitative prediction of the locations where cavitation appears. For a quantitative prediction, results with mass transfer are more realistic. It is interesting to note that the maximum vapor mass fraction is not very large (less than 0.13 ). The pressure level in the cavitation pocket is quite different: $0.4 \mathrm{~atm}$ with mass transfer, corresponding to the saturation pressure, to be compared with $10^{-3}$ atm in absence of mass transfer.

\subsubsection{High pressure fuel injector.}

The second situation under study consists in a nozzle where liquid fuel, from a high pressure chamber is injected into another chamber at atmospheric pressure. The high pressure tank (1000 atm) is filled with liquid dodecane at density $570 \mathrm{~kg} / \mathrm{m}^{3}$, corresponding to the temperature $T=640 K$ (lower than the critical temperature). As previously a weak volume fraction of vapor $\left(\alpha_{v}=10^{-4}\right)$ is present in the liquid. The initial conditions consist in an initial discontinuity between liquid dodecane at $1000 \mathrm{bar}$ and $570 \mathrm{~kg} / \mathrm{m}^{3}$ and its vapor at atmospheric pressure. Results are shown in figure 23 at times $t=24 \mu s$, $t=6(0 \mu s, t=120 \mu s, t=6(0) \mu s$. On the left. computed results without mass transfer are presented, and on the right those including evaporation. As in the preceding example, the results are qualitatively similar. But quantitative differences are present regarding flow variables.

It is interesting to note that only slight differences in cavitation pocket size are present. Two-phase nozzle flows have been used in the past to determine mass transfer rate in non-equilibrium two-phase flow models. Mass transfer rate was adjusted in order to match the cavitation pocket size. The present computations show that such method is inappropriate. Indeed, cavitation pocket size has a too weak dependence regarding mass transfer. Mechanical effects alone are able to produce significant cavitation pockets. 

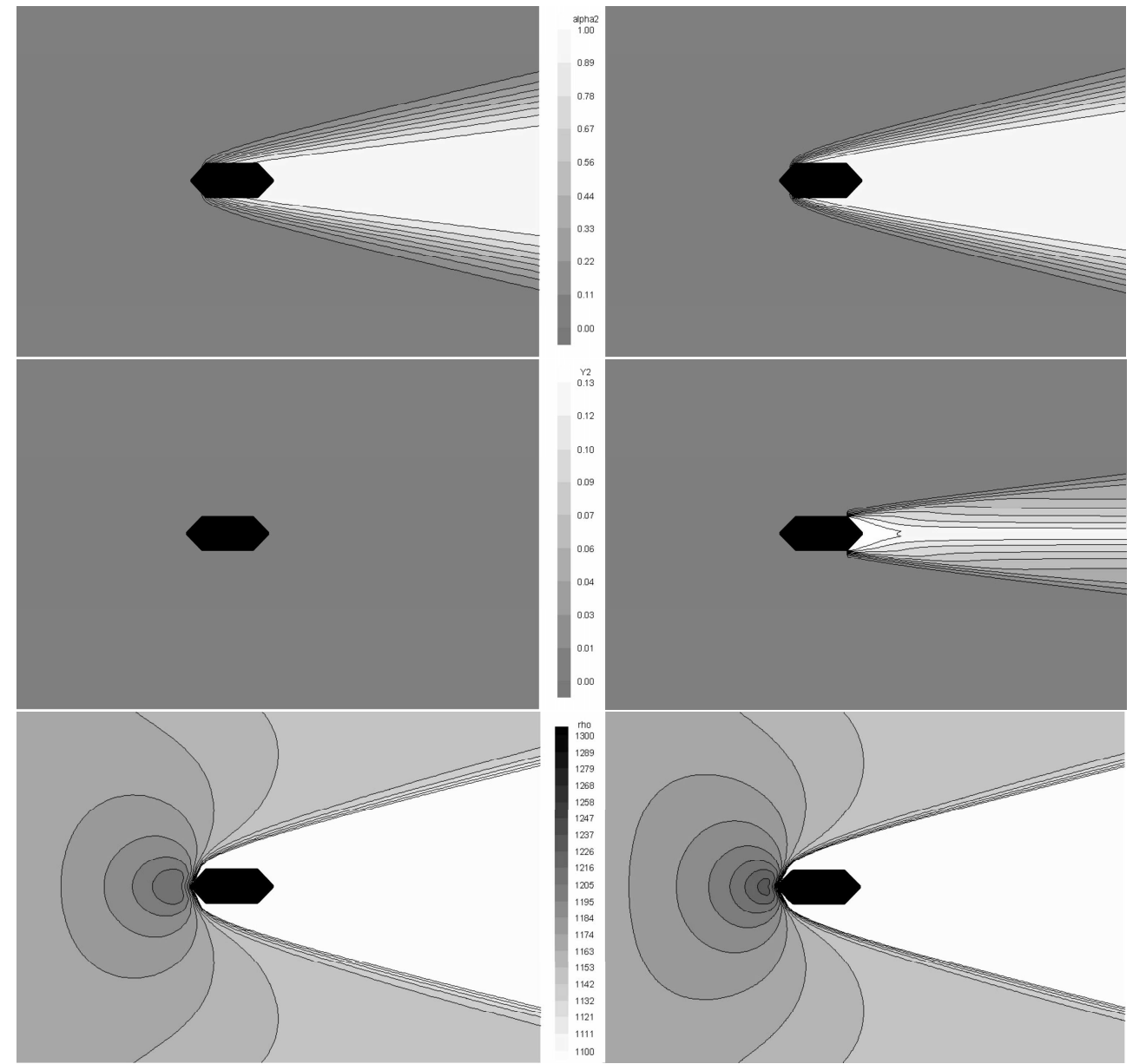

Figur. 21. High velocity underwater projectile. On the left, the solution obtained without heat and mass transfer and on the right the solution with the new thermo-chemical solver. Solutions are shown at steady state. From top to bottom, contours of : vapor volume fraction, vapor mass fraction and mixture density. White regions represent from top to bottom respectively: regions where vapor volume fraction is closed to 1 . regions where vapor mass fraction becomes non negligible (0.1) and regions where mixture density is lower than the density at infinity. 


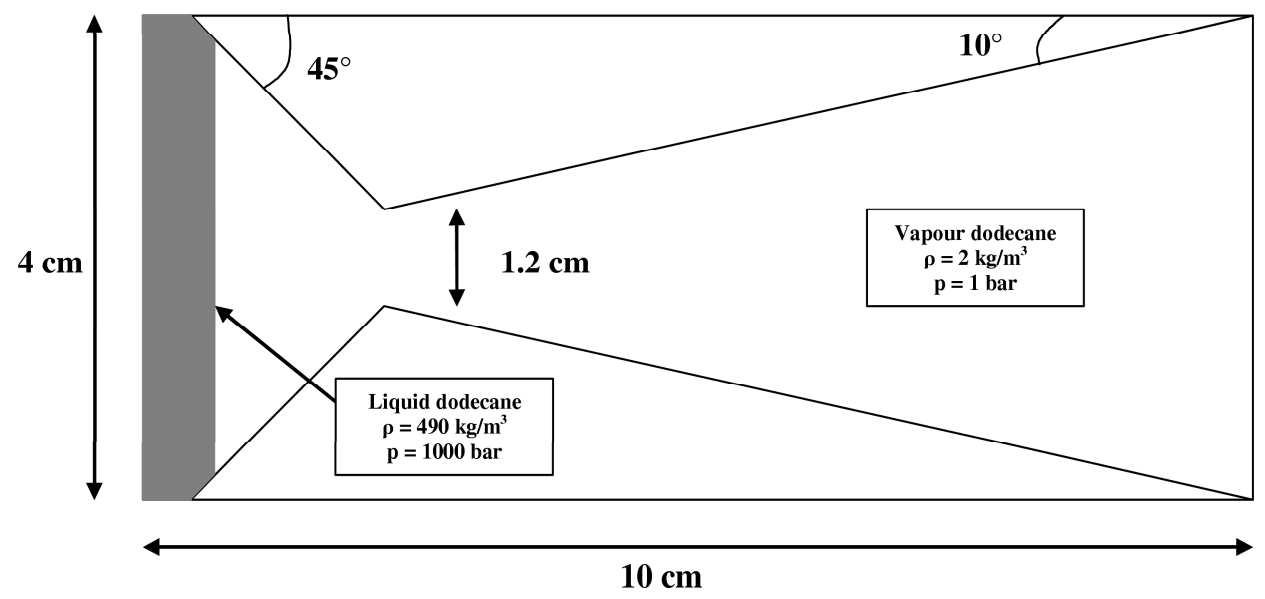

Figur.e 22. Initial configuration of a high pressure fuel injector
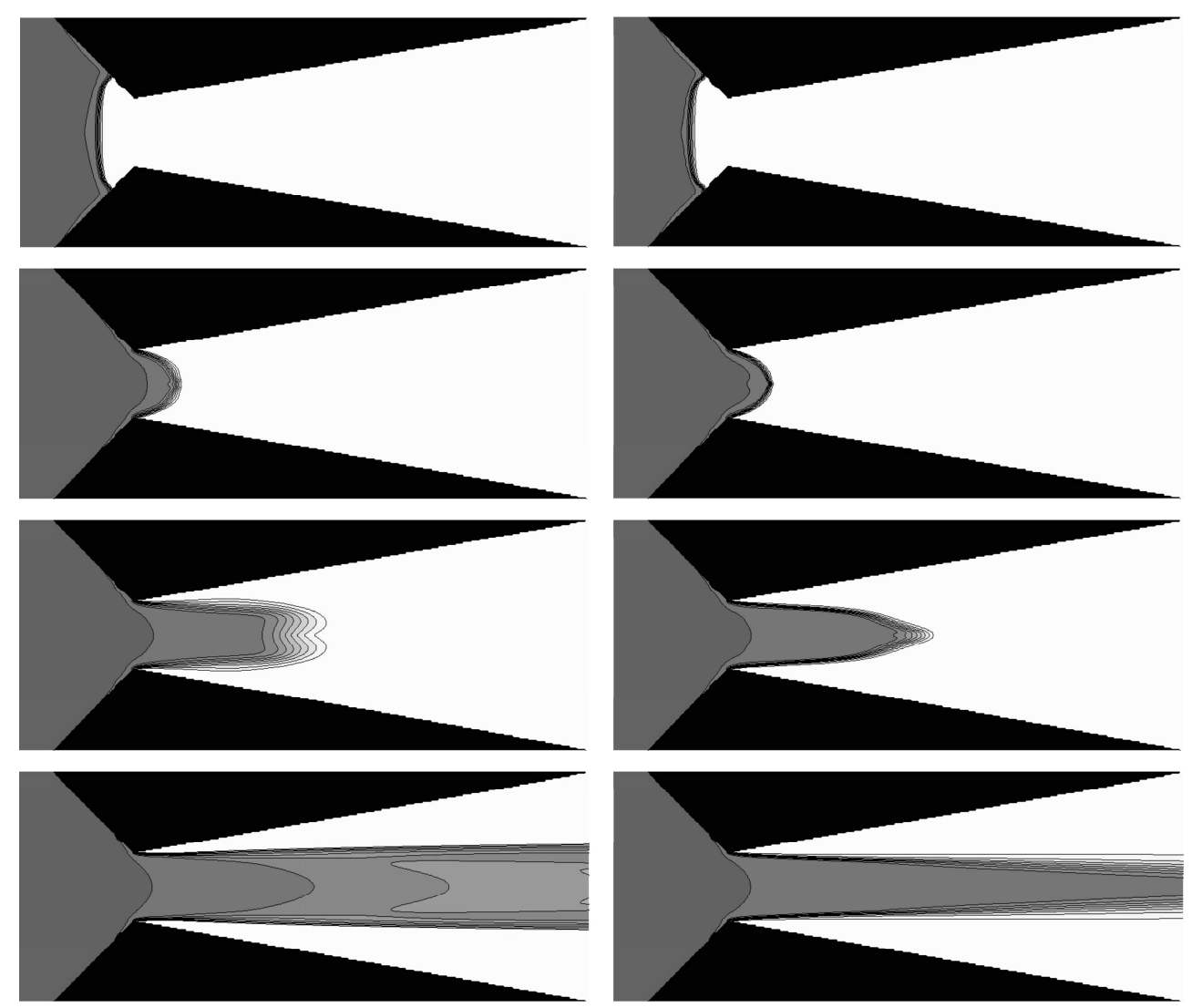

Figure 23. Flow in a high pressure fuel injector. Mixture density contours are shown at times $t=24 \mu \mathrm{s}, t=60 \mu \mathrm{s}, t=120 \mu \mathrm{s}$ and $t=600 \mu \mathrm{s}$. On the left. the solution is obtained without heat and mass transfers and on the right the solution is obtained with the thermo-chemical solver. 


\section{Conclusions}

A new hyperbolic compressible flow model has been built for the computation of cavitating flows. Relaxation effects are modeled and are able to connect the non equilibrium flow model to the mixture Euler model when thermodynamic equilibrium is reached. The connection occurs through rarefaction waves where mass transfer occurs. The overall model is able to predict dynamic evaporation waves as well as interfaces of simple contact separating non-miscible phases.

The model analysis shows that the Chapmann Jouguet kinetic relation for the dynamics of evaporation fronts (Chaves 1984: Thompson et al. 1987) is recovered as an expansion wave of the present model in the limit of stiff thermal and chemical relaxation. Numerical examples show that the model is able to predict complex flow pattern in one and two dimensions.

\section{Appendix A. Determination of equilibrium speed of sound.}

From the definition of mixture internal energy, we have:

$$
e=\left(\alpha_{1} \rho_{1} e_{1}+\alpha_{2} \rho_{2} e_{2}\right) / \rho
$$

When the mixture evolves under thermodynamic equilibrium, the temperature is linked to the pressure (4.3). Each thermodynamic variable can thus be expressed as a function of pressure only: $e_{k}(p)$ and $\rho_{k}(p)$. Equation (A 1) can be written as:

$$
e(\rho, p)=\frac{1}{\rho}\left(\rho_{1} e_{1}+\frac{\rho-\rho_{1}}{\rho_{2}-\rho_{1}}\left(\rho_{2} e_{2}-\rho_{1} e_{1}\right)\right) .
$$

The mixture speed of sound definition reads:

$$
c^{2}=\left(\frac{\partial p}{\partial \rho}\right)_{s}=\frac{\frac{p}{\rho^{2}}-\left(\frac{\partial e}{\partial \rho}\right)_{p}}{\left(\frac{\partial e}{\partial p}\right)_{\rho}}
$$

Differenciating $e$ wit respect to $\rho$ and $p$, we obtain:

$$
\frac{1}{\rho c_{e q}^{2}}=\frac{-\frac{d \rho_{1}}{d p} \alpha_{1} \rho_{2}\left(\frac{e_{2}-e_{1}}{\rho_{2}-\rho_{1}}\right)-\frac{d \rho_{2}}{d p} \alpha_{2} \rho_{1}\left(\frac{e_{2}-e_{1}}{\rho_{2}-\rho_{1}}\right)+\frac{d e_{1}}{d p} \alpha_{1} \rho_{1}+\frac{d e_{2}}{d p} \alpha_{2} \rho_{2}}{p-\rho_{1} \rho_{2}\left(\frac{e_{2}-e_{1}}{\rho_{2}-\rho_{1}}\right)} .
$$

This sound speed is the equilibrium one as (4.3) has been used. We now use the Gibbs relation for each phase:

$$
\forall k=1,2 \quad \frac{d e_{k}}{d p}=T \frac{d s_{k}}{d p}+\frac{p}{\rho_{k}^{2}} \frac{d \rho_{k}}{d p}
$$

Noting that

$$
\text { for } k=1,2 \quad \frac{d \rho_{k}}{d p}=\left(\frac{\partial \rho_{k}}{\partial p}\right)_{s_{k}}+\left(\frac{\partial \rho_{k}}{\partial s_{k}}\right)_{p} \frac{d s_{k}}{d p},
$$

we obtain the mixture equilibrium speed of sound formulation (5.20):

$$
\frac{1}{\rho c_{e q}^{2}}=\frac{\alpha_{1}}{\rho_{1} c_{1}^{2}}+\frac{\alpha_{2}}{\rho_{2} c_{2}^{2}}+T\left[\frac{\alpha_{1} \rho_{1}}{C_{p, v}}\left(\frac{d s_{1}}{d p}\right)^{2}+\frac{\alpha_{2} \rho_{2}}{C_{p, l}}\left(\frac{d s_{2}}{d p}\right)^{2}\right] .
$$




\section{Appendix B. Coefficients A, B, A' and B' for thermo chemical solver.}

In order to determine heat and mass transfer terms (6.6), the four coefficients A. B. A' and B' have to be calculated at time $t^{n}$. Here, we provide expressions of these coefficients in the context of SG EOS. They write:

$$
\begin{aligned}
& A=-\left(C_{1}-C_{2}\right) \rho c^{2}\left(\frac{\Gamma_{1}}{\rho_{1} c_{1}^{2}}-\frac{\Gamma_{2}}{\rho_{2} c_{2}^{2}}\right)+\frac{1}{C_{v, 1} \gamma_{1} \alpha_{1} \rho_{1}}+\frac{1}{C_{v, 2} \gamma_{2} \alpha_{2} \rho_{2}} \\
& B=-\left(C_{1}-C_{2}\right) \rho\left[\left(\frac{\rho c^{2}}{\rho_{1}}-\Gamma h_{1}\right)-\left(\frac{\rho c^{2}}{\rho_{2}}-\Gamma h_{2}\right)\right] \\
& -\rho \Gamma\left(h_{1}-h_{2}\right)\left(\frac{1}{C_{v, 1} \gamma_{1} \Gamma_{1} \rho_{1}}-\frac{1}{C_{v, 2} \gamma_{2} \Gamma_{2} \rho_{2}}\right) \\
& A^{\prime}=\left(D_{1} C_{1}-D_{2} C_{2}\right) \rho c^{2}\left(\frac{\Gamma_{1}}{\rho_{1} c_{1}^{2}}-\frac{\Gamma_{2}}{\rho_{2} c_{2}^{2}}\right) \\
& -\left(1+\frac{D_{1}}{C_{v, 1} \gamma_{1}}\right) \frac{1}{\alpha_{1} \rho_{1}}-\left(1+\frac{D_{2}}{C_{v, 2} \gamma_{2}}\right) \frac{1}{\alpha_{2} \rho_{2}} \\
& B^{\prime}=\left(D_{1} C_{1}-D_{2} C_{2}\right) \rho\left[\left(\frac{\rho c^{2}}{\rho_{1}}-\Gamma h_{1}\right)-\left(\frac{\rho c^{2}}{\rho_{2}}-\Gamma h_{2}\right)\right] \\
& +\rho \Gamma\left(h_{1}-h_{2}\right)\left[\left(1+\frac{D_{1}}{C_{v, 1} \gamma_{1}}\right) \frac{1}{\Gamma_{1} \rho_{1}}+\left(1+\frac{D_{2}}{C_{v, 2} \gamma_{2}}\right) \frac{1}{\Gamma_{2} \rho_{2}}\right]
\end{aligned}
$$

where the coefficients $C_{k}$ and $D_{k}$ are given for each phase $k$ by:

$$
\begin{aligned}
C_{k} & =\frac{\left(1-\gamma_{k}\right) T_{k}}{\gamma_{k}\left(p+p_{\infty, k}\right)} \\
D_{k} & =\frac{q_{k}-g_{k}}{T_{k}} .
\end{aligned}
$$

\section{REFERENCES}

Abgrall, R. \& SAURel. R. 2003 Discrete equations for physical and numerical compressible multiphase mixtures. Journal of Computational Physics 186(2), 361-396.

Baer. M.R. \& Nunziato. J.W. 1986 A two-phase mixture theory for the deflagration-todetonation transition (DDT) in reactive granular materials. Int. J. of Multiphase Flows 12, 861-88.9.

Butler, P.B.. LAmbeck. M.F. \&. Krier. H. 1982 Modeling of shock development and transition to detonation initiated by burning in porous propellant beds. Comb. Flame 46. 75-93.

CAHn. J.W. \&. Hilliard, J.E. 1958 Free energy of a nonuniform system i: Interfacial free. energy. I. Chem. Physics 28.

Chaves, H. 1984 Changes of phase and waves on depressurization of liquids with high specific hea.t. Mitt. Max-Planck-Institut f. Strmungsforschung $\mathbf{7 7 .}$

Chinnayya, A., Daniel, F. \& Saurel, R. 2004 Computation od detonation waves in heterogeneous energetic materials. Journal of Computational Physics 196. 490-538.

Delhaye. .J.M. \&. Boure, .J.A. 1982 General Equations and Two-Phase Flow Modeling. In: Handbook of Multiphase Systems. In: Handbook of Multiphase Systems, edited by G. Hestroni, Hemisphere Publishing Corp., New-York.

Faucher, E.. Herard, J.M., Barret, M. \& Toulemonde, C. 2000 Computation of flashing flows in variable cross section ducts. Int. J. Comp. Fluid Dynamics $\mathbf{1 3}$

Frost, D.L., LeE, J.H.S. \& Ciccarelli, G. 1991 The use of hugoniot analysis for the propagation of vapor explosion waves. Shock Waves 1. 99-110. 
Grolmes, M.A. \& FAuske, H.K. 1974 Axial propagation of free surface boiling into superheated liquids in vertical tubes. Heat transfer 4, 30-34.

Hill. L.G. \&. Sturtevant. B. 1990) An experimental study of evaporation waves in a superheated liquid. In Adiabatic Waves in Liquid-Vapor System (ed. G.E.A. Meier and P.A. Thompson), Springer pp. 25-37.

Ishiı, M. 1975 Thermo-Fluid Dynamic Theory of Two-Phase Flouı.. Eyrolles, Paris.

Jamet, D., Lebaigue. O.. Coutris, N. \& Delhaye, .J.M. 2001 The second gradient method for the direct numerical simulation of liquid-vapor flows with phase change. J. Comp. Physics 169, 624-651.

Kapila, A.: Menikoff, R.. Bdzil. J., Son, S. \& Stewart, D. 2001 Two-phase modeling of DDT in granular materials: reduced equations. Physics of Fluids 13. 3002-3024.

Kurschat, Th., Chaves, H. \&. Meier, G.E.A. 1992 Complete adiabatic evaporation of highly superheated liquid jets. ,J. Fluid Mech. 236, 43-59.

t.f Metayer. O.. Massoni, .J. \&. Saurel, R.. 2004 Elaborating equations of state of a liquid and its vapor for two-phase flow models. Int. J. of Thermal Sciences 43. 265-276.

I. Me Metayer. O.. Massoni, .J. \&. SAurel. R. 2005 Modeling evaporation fronts with reactive Riemann solvers. Journal of Computational Physics 205, 567-610.

LIOU, M.S. \& EdWARDS, J.R. 1999 AUSM Schemes and Extensions for Low Mach and Multiphase Flows. 30th Computational Fluid Dynamics, March 8-12, Lect. Series 1999-03, Von Karman Institute for Fluid Dynamics.

Menikoff, R. \&. Plohr, B..J. 1989 The Riemann problem for fluid flow of real materials. Rev. Mod. Physics 61, 75-130).

Murrone, A. \& Guillard, H. 2004 A five equations reduced model for compressible two-phase flow problems. Journal of Computational Physics 202. 664-698.

Oldenbourg, R. 1989 Properties of water and steam in si-11nits. Springer .

Perigaud, G. \& SAurel, R. 2005 A compressible flow model with capillary effects. J. Comp. Phys. 209, 139-178.

Petitpas. F.. Franquet. E.. Saurel. R. \& If. Metayer. O. 2007 A relaxation-projection method for compressible flows. Part 2: Artificial heat exchange for multiphase shocks. Journal of Computational Physics 225(2), 2214-2248.

Reinke. P. \& YADIGARoglu, G. 2001 Explosive vaporization of superheated liquids by boiling fronts. Int. J. of Multiphase Flows 27, 1487-1516.

Saurel, R. \& ABgrall. R. 1999 A multiphase Godunov method for compressible multifluid and multiphase flows. Journal of Computational Physics 150, 425-467.

Saurel, R., Cocchi, .J.P. \&. Butler, P.B. 1999 A numerical study of cavitation in the wake of a hypervelocity underwater projectile. J. of Propulsion and Power 15.

Saurel, R.. Franquet, E.. Daniel. F. \& If. Metayer. O. $2007 a$ A relaxation-projection method for compressible flows. Part 1: The numerical equation of state for the Euler Equations. Journal of Computational Physics 223. 822-845.

Saurel, R.. Gavrilyuk. S. \& Renaud, F. 2003 A multiphase model with internal degrees of freedom: application to shock-bubble interaction. Journal of Fluid Mechanics 495, 283-321.

SAurel, R. \& Le Metayer: O. 2001 A multiphase model for compressible flows with interfaces: shocks, detonation waves and cavitation. Journal of Fluid Mechanics 431, 239-271.

Saurel, R., If. Metayer, O., Massoni, .J. \& Gavrilyuk, S. 2007 b Shock jump relations for multiphase mixtures with stiff mechanical relaxation. Shock Waves 16. 209-232.

Simoes-Moreira, J.R. 1994 Adiabatic Evaporation Waves. PhD Thesis, Rensselaer Polytechnic Institute, Troy.

Simoes-MoreirA. J.R. \& Shepherd, J.E. 1999 Evaporation waves in superheated dodecane. J. Fluid Mech. 382, 63-86.

Thompson. P.A., Chaves, H., Meier, G.E.A, Kim. Y.G. \& Speckmann, H.D. 1987 Wave splitting in a fluid of large heat capacity. J. Fluid. Mech. 185. 385-414.

Thompson, P.A. \&. Lambrakis, K.C. 1973 Negative shock waves. Journal of Fluid Mechanics 60, $187-208$.

Wood: A.B. 1930 A textbook of sound. G. Bell and Sons LTD, London . 